\title{
Synthesis, crystal and magnetic structure of the spin-chain compound $\mathrm{Ag}_{2} \mathrm{RuO}_{4}$
}

\author{
Beluvalli E. Prasad, ${ }^{1}$ Surasree Sadhukhan, ${ }^{2}$ Thomas C. Hansen $\odot,{ }^{3}$

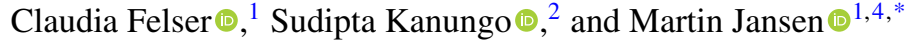 \\ ${ }^{1}$ Max-Planck-Institut für Chemische Physik fester Stoffe, Nöthnitzer Strasse 40, 01187 Dresden, Germany \\ ${ }^{2}$ School of Physical Sciences, Indian Institute of Technology Goa, 403401 Goa, India \\ ${ }^{3}$ Institut Max von Laue-Paul Langevin, 71 Avenue des Martyrs, 38000 Grenoble, France \\ ${ }^{4}$ Max-Planck-Institut für Festkörperforschung, Heisenbergstrasse 1, 70569 Stuttgart, Germany
}

(Received 11 October 2019; accepted 2 January 2020; published 28 February 2020)

\begin{abstract}
We report synthesis and crystal structure refinement of $\mathrm{Ag}_{2} \mathrm{RuO}_{4}$, followed by combined analysis of its physical properties through bulk experimental tools (magnetic susceptibility, electron transport, and heat capacity measurements), a microscopic experimental tool (temperature dependent neutron diffraction), and $a b$ initio first-principles calculations. We observe a rather unique $\left(\mathrm{RuO}_{3 / 1} \mathrm{O}_{2 / 2}\right)_{\mathrm{n}}$ polyoxoanion, where $\mathrm{Ru}$ is in a distorted trigonal bipyramidal coordination by oxygen. $\mathrm{The}^{\mathrm{RuO}} 5$ polyhedra are linked via the apical oxygen atoms to form chains extending along the crystallographic $a$ axis. Crystal structure, magnetization, and $a b$ initio calculations indicate that $\mathrm{Ru}$ is in the +6 oxidation state with a nominal valence electron configuration of $4 d^{2}$. Bulk magnetization, specific-heat, and neutron-diffraction measurements provide clear indication of an antiferromagnetic transition around $75 \mathrm{~K}$ with moderate spin canting in the order of $30^{\circ}$ with respect to the $c$ axis. The neutron-diffraction results as well as the density functional theory based first-principles calculations of exchange interactions revealed that the strong intrachain interaction is predominantly of ferromagnetic (FM) type, and that this spin order along the chains couples with the neighboring chains through comparatively weak FM and antiferromagnetic interactions. Notably, the Landé $g$ factor is found to be 1.8 (with an infinite chain model and even a simple Curie-Weiss approach), away from the ideal value of 2 , due to the low dimensionality of the $\mathrm{Ru} / \mathrm{O}$ substructure.
\end{abstract}

DOI: $10.1103 /$ PhysRevMaterials.4.024418

\section{INTRODUCTION}

Low-dimensional quantum magnets constitute a highly rated and investigated topic in the condensed matter community due to their versatile novel features [1], such as a Haldane gap [2] in the $S=1$ spin-chain compound $\mathrm{Ni}\left(\mathrm{C}_{5} \mathrm{H}_{14} \mathrm{~N}_{2}\right)_{2} \mathrm{~N}_{3}\left(\mathrm{PF}_{6}\right)$ [3], gapless magnetic excitation in $S=1 / 2$ Heisenberg antiferromagnetic (AFM) chains [4], spin dimerization, unconventional spin excitations, quantum criticality [5,6], a spin-Peierls transition in $\mathrm{CeCuGe}_{3}$ [7], a quantum spin-liquid state in $\kappa$-(BEDT-TTF $)_{2} \mathrm{Cu}_{2}(\mathrm{CN})_{3}[8,9]$, or a bilayer magnet in $\mathrm{Ca}_{10} \mathrm{Cr}_{7} \mathrm{O}_{28}$ [10]. Although these materials are structurally three dimensional, the effective low dimensionality in this context arises because of the strong directional character of the crystal structure, where unique arrangements of the constituent magnetic ions induce anisotropic electronic and magnetic interactions. In the past, the main focus was on $3 d$ transition-metal (TM) based systems. More recently, ternary oxides with heavy TMs ( $4 d$ and $5 d$ ) have attracted particular attention due to the possibilities of the intimate interplay between electron-electron correlation, spin-orbit coupling (SOC), and crystal-field splitting,

\footnotetext{
*Corresponding author: m.jansen@fkf.mpg.de
}

Published by the American Physical Society under the terms of the Creative Commons Attribution 4.0 International license. Further distribution of this work must maintain attribution to the author(s) and the published article's title, journal citation, and DOI. Open access publication funded by the Max Planck Society. which are in a comparable energy scale for $4 d$ and $5 d$ TMs. Whereas the $5 d$ iridates have been studied extensively in the context of low-dimensional magnets in the form of Heisenberg-Kitaev spin liquids $[11,12]$ and $J_{\text {eff }}=1 / 2$ spin-orbit coupled Mott insulators in layered iridates [13,14], $4 d$ TM based ternary oxides are comparatively less investigated in this respect. Nonetheless, among the $4 d$ TMO's, ruthenates exhibit extraordinary properties in terms of both structural aspects as well as electronic and magnetic responses. The binary oxide $\mathrm{RuO}_{2}$ [15] is a metallic conductor with Pauli paramagnetism; the perovskite-type ternary $\mathrm{SrRuO}_{3}, \mathrm{CaRuO}_{3}$, and $\mathrm{BaRuO}_{3}$ show metallic conduction with ferromagnetism below $165 \mathrm{~K}$, are paramagnetic metals down to $4 \mathrm{~K}$ with AFM interactions, and adopt varied magnetic states depending on the synthesis conditions [16-21]; while $\mathrm{Sr}_{2} \mathrm{RuO}_{4}$ [22] exhibits unconventional $p$-type superconductivity. In the context of low-dimensional magnets ruthenates have come into the picture with the discovery of layered $\boldsymbol{\alpha}-\mathrm{RuCl}_{3}[23,24]$ as a spinliquid system, followed by one-dimensional (1D) $\mathrm{Li}_{3} \mathrm{RuO}_{4}$ [25,26] and two-dimensional $\mathrm{Li}_{2} \mathrm{RuO}_{3}$ [27-29] materials.

We are interested in new low-dimensional topologies of ruthenates with different arrangements of magnetic Ru ions in higher oxidation states, compared to $\mathrm{Ru}^{+4}$ or $\mathrm{Ru}^{+5}$ as discussed above. Such phases may give rise to quantum fluctuations due to small spin values and to various interesting ground-state magnetic properties. However, from the synthesis point of view achieving such a goal is not trivial. Our strategy is to play with the charge compensating counterions in ternary oxoruthenates. Here, the alkali-metal and alkaline-earth cations are commonly first choice, due to 
two complexions, namely, by the expectation that the high oxide basicities [30,31] of such binary oxides would foster solid-state reactivity, and that the "innocent" character of the alkali-metal and alkaline-earth cations would not impose a steric strain to the crystal structures forming and would demand nothing but an adequate share of space. Given such a matrix, the second constituting cation would be given freedom to develop a large variety of compositions, valence states, and electronic structures. The choice in alternative monovalent cations-which are essentially $\mathrm{Cu}^{1+}, \mathrm{Ag}^{1+}$, or $\mathrm{Tl}^{1+}$-is but scarce, and each of them poses particular challenges when involved in solid-state syntheses. For silver(I) oxide, its low thermal stability constitutes a crucial impediment. This handicap, however, has been overcome by maintaining a sufficiently high oxygen pressure during solid-state reaction of couples of respective binary oxides [32]. Using autoclaves, capable of enduring oxygen pressures and temperatures of about $0.7 \mathrm{GPa}$ and $700^{\circ} \mathrm{C}$, has opened a particularly valuable parameter window for reacting silver(I) oxide with virtually any binary oxide in the periodic table, thus enabling researchers to explore wide areas for unknown materials experimentally.

Since the effective radii of $\mathrm{Ag}^{1+}$ and of $\mathrm{Na}^{1+}$ are comparable in size, one would expect to encounter lots of similarities between silver and sodium oxometallates. However, in contrast, most of the time for the same composition distinctly different structures and properties were revealed. Of course, the inclination of $\mathrm{Ag}^{1+}$ to form more covalent bonds to oxygen and to strive for low coordination numbers is relevant in this context, but in addition new appreciably structure directing characteristics have been disclosed. Noteworthy, the equally charged silver cations tend to aggregate to clusterlike extended assemblies with interatomic separations in the same range as found for elemental silver, and even below [33]. Such weakly attractive (metallophilic) $d^{10}-d^{10}$ interactions have a significant effect on the structural evolutions [34,35], and give rise to special transport properties [36] and visible light absorption [37].

Utilizing high-oxygen-pressure and hydrothermal synthesis techniques, we started to explore $\mathrm{Ag}_{2} \mathrm{O} / \mathrm{RuO}_{x}$ systems, which has resulted in the discovery of several silver ruthenates. Among them, $\mathrm{AgRuO}_{3}$ shows strong antiferromagnetic exchange coupling on a $\left[\mathrm{RuO}_{3}\right]$ honeycomb lattice [38], $\alpha-\mathrm{Ag}_{3} \mathrm{RuO}_{4}$ forms a $1 \mathrm{D}$ polyoxoanion of edge-sharing octahedra [39], while the $\beta$ polymorph features an oligomeric oxoanion [40]. The latter representative is kind of exceptional in as much it is closely related to the sodium analog, containing the same tetrameric oxoanion [41-43], showing frustrated-magnetic exchange coupling within the tetrameric spin cluster $[40,43]$.

Here, we report on synthesis, crystal structure, and details of magnetic structure of $\mathrm{Ag}_{2} \mathrm{RuO}_{4}$, which likewise is related to the sodium analog [44-47]. $\mathrm{Ag}_{2} \mathrm{RuO}_{4}$ was mentioned for the first time back in 1967, when Nowogrocki, working on ruthenates(VI) and ruthenates(VII), signalized the synthesis of the title compound by precipitation out of an aqueous solution of potassium per-ruthenate(VII) with a soluble salt of silver(I) [48]. Samples prepared along this route show the same crystal structure as found for the title compound.

We investigated $\mathrm{Ag}_{2} \mathrm{RuO}_{4}$ through macroscopic measurements like magnetic susceptibility, electron transport, and heat capacity measurements, a microscopic experimental tool of temperature-dependent neutron-diffraction and $a b$ initio first-principles calculations. The analysis shows a chain-type polyoxoanion consisting of trigonal bipyramidal $\left[\mathrm{RuO}_{5}\right]$ primary building units sharing the apical vertices, forming chains along the crystallographic $a$ axis. Thus, $\mathrm{Ag}_{2} \mathrm{RuO}_{4}$ belongs to the class of low-dimensional quantum magnets. It shows a clear AFM transition at around $75 \mathrm{~K}$, with moderate spin canting. Electronic structure calculations revealed that strong intrachain interaction among the $\mathrm{Ru}^{+6}\left(4 d^{2}\right)$ is predominantly ferromagnetic. Remarkably, the sister compound of $\mathrm{Ag}_{2} \mathrm{RuO}_{4}$, i.e., $\mathrm{Na}_{2} \mathrm{RuO}_{4}$, although structurally quite similar, shows a different interesting anisotropic quasi-1D nature magnetic structure $[45,47]$.

\section{EXPERIMENTAL}

\section{A. Synthesis}

The title compound was synthesized from $\mathrm{Ag}_{2} \mathrm{O}$ (freshly precipitated with $\mathrm{KOH}$ from an aqueous solution of $\mathrm{AgNO}_{3}$ ) and $\mathrm{KRuO}_{4}$ (Alfa Aesar 97\%) by applying hydrothermal conditions using a Teflon-lined stainless-steel autoclave. For a typical batch of about $150 \mathrm{mg}, \mathrm{Ag}_{2} \mathrm{O}$ and $\mathrm{KRuO}_{4}$ in the required molar ratio of 1:1 were added to $8 \mathrm{ml}$ of deionized water, the mixture was stirred subsequently for $10-15 \mathrm{~min}$, placed in a $25-\mathrm{ml}$ autoclave, and heated to $140-150{ }^{\circ} \mathrm{C}$ for 48-72 h. After switching off the heating source, the autoclave was allowed to cool to room temperature. The product formed was filtered off, washed with deionized water and ethanol, and finally dried in a desiccator.

\section{B. Thermal analysis}

The thermal stability of the title compound was monitored by thermogravimetric analysis (TGA)/differential thermal analysis (DTA) (NETZSCH STA $449^{\circ} \mathrm{C}$, argon atmosphere, flow rate $100 \mathrm{ml} / \mathrm{min}$, heating rate $10 \mathrm{~K} / \mathrm{min}$ ).

\section{X-ray and neutron powder diffraction}

A powder pattern of the sample was recorded by $\mathrm{x}$-ray diffraction (Huber, $\mathrm{G} 670, \mathrm{Cu}-K \alpha_{1}$ radiation, $\lambda=1.54056 \AA$ ). Rietveld refinement of the x-ray powder pattern was carried out using the program TOPAS-V4.2.0.2 [49] (Fig. 1). Neutron powder-diffraction experiments were performed at the high intensity diffractometer D20 at ILL, Grenoble [50]. For confirmation of the nuclear structure, a high-resolution configuration was used with a wavelength of 1.48 A obtained from the (335) reflection of a germanium monochromator at $118^{\circ}$ takeoff angle. Patterns were acquired at room temperature (296 K), just above the magnetic transition temperature ( $76 \mathrm{~K}$ ), and the cryostat's base temperature of $1.8 \mathrm{~K}$.

For the precise, unambiguous determination of the magnetic structure a high intensity configuration was used with a wavelength of $2.41 \AA$ from the (002) reflection of a highly oriented pyrolytic graphite monochromator. A thermodiffractometry experiment was performed in this configuration with data acquisitions of 2 min each with the stationary $153.6^{\circ}$ covering position sensitive detector. The temperature was raised from 1.8 to $76 \mathrm{~K}$ at a rate of $2.5 \mathrm{~K}$ per data acquisition, and another one from $\approx 1.8$ to $113.4 \mathrm{~K}$ at a rate of $\approx 1.1 \mathrm{~K}$ 


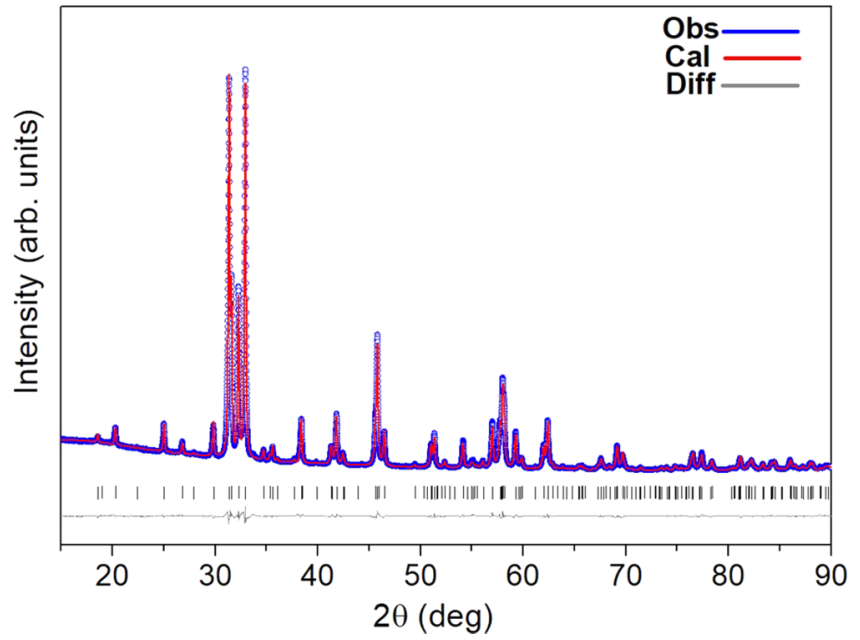

FIG. 1. Rietveld fit of the PXRD pattern of $\mathrm{Ag}_{2} \mathrm{RuO}_{4}$.

per data acquisition, in order to follow the temperature dependence of the magnetic moment. Particularly long acquisitions were performed at 1.8 and $76 \mathrm{~K}$ (just above the magnetic ordering temperature), with $10 \mathrm{~h}$ of counting time each, using a detector scan for more precision (excluding parasitic signals and faulty detector efficiencies), two scans for each temperature, covering $3^{\circ}$ with a step width of $0.05^{\circ}$ with 5 min counting time per step.

\section{Measurement of physical properties}

Magnetization was measured in an MPMS-XL7 magnetometer (Quantum Design) between 1.8 and $400 \mathrm{~K}$. The electrical resistivity was recorded on a powder sample pressed in a sapphire die cell with four Pt contacts using the van der Pauw method and reversing direct current. Heat capacity was determined in the temperature range 1.8-300 K (HC option, PPMS, Quantum Design).

\section{E. Computational methods}

In order to characterize the electronic structure and magnetic properties, density functional theory (DFT) based investigations were carried out using the low-temperature $(1.8 \mathrm{~K})$ neutron-diffraction crystal structure, within the planewave basis set based on a pseudopotential framework as implemented in the Vienna $a b$ initio simulation package VASP $[51,52]$. The exchange-correlation functional was chosen to be the generalized gradient approximation (GGA) following the Perdew-Burke-Ernzerhof prescription [53]. The missing electronic correlation beyond the standard GGA, which is necessary for a transition-metal ion such as $\mathrm{Ru}$, was accounted for by using Hubbard on-site coulomb correlation as implemented in the GGA $+U$ method [54,55], with a choice of the Hubbard $(U=2.9 \mathrm{eV})$ and Hund's coupling $\left(J_{\mathrm{H}}=0.9 \mathrm{eV}\right)$ at the $\mathrm{Ru}$ site, thus considering values commonly applied for ruthenate compounds. The results shown are for $U=$ $2.9 \mathrm{eV}$ and $J_{\mathrm{H}}=0.9 \mathrm{eV}$. We also crosschecked the influence on our results by varying the $U_{\text {eff }}$ values in the range of 1 to $4 \mathrm{eV}$. We optimized the experimental crystal structure by relaxing the position of each atom up to $0.005 \mathrm{eV} / \AA$ in the

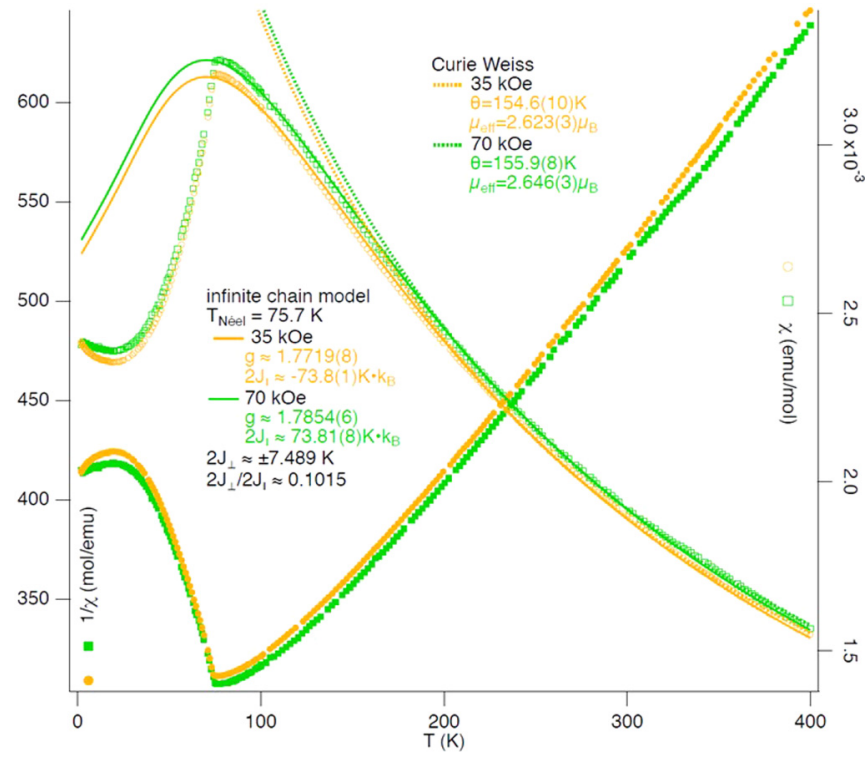

FIG. 2. Magnetic susceptibility (empty squares and circles) and inverse susceptibility (filled squares and circles) measured in an applied field of $70 \mathrm{kOe}$ (green squares) and $35 \mathrm{kOe}$ (orange circles). The solid lines represent fits of the data in the range from 75 to $400 \mathrm{~K}$ calculated with a model for isolated chains of classical spins with refined parameters $2 J_{\|} \approx-74 \mathrm{~K}$ and $g \approx 1.8$. The dotted lines represent fits with the Curie-Weiss law. All data were recorded in FC mode.

unit cell, keeping the lattice parameters the same as obtained from the neutron-diffraction measurement. For self-consistent calculations we used an electronic convergence criterion of $10^{-7} \mathrm{eV}$ and a $6 \times 6 \times 6$ mesh division for the Brillouin-zone integration.

\section{RESULTS}

\section{A. Synthesis and bulk properties}

$\mathrm{Ag}_{2} \mathrm{RuO}_{4}$ was synthesized along an improved synthetic route, namely, from $\mathrm{Ag}_{2} \mathrm{O}$ and $\mathrm{KRuO}_{4}$, applying hydrothermal conditions. As evidenced by powder x-ray diffraction (PXRD) (Fig. 1), the solid formed is single phased and of good crystallinity. The shiny-black product is insensitive to air and moisture, and starts decomposing at $360^{\circ} \mathrm{C}$, according to simultaneous DTA/TGA (Fig. S1 in Supplemental Material [56]). The weight loss (8.4\%) during the first step of decomposition corresponds to the mass of two equivalents of oxygen. The solid residual was characterized by PXRD, and consists of metallic silver and $\mathrm{RuO}_{2}$.

The susceptibility of the sample measured as a function of temperature in an applied field of 70 and $35 \mathrm{kOe}$ (Fig. S2 [56]) indicates AFM exchange coupling with magnetic ordering occurring at $T_{\mathrm{N}}=75.4 \mathrm{~K}$. The effective magnetic moment calculated by fitting the Curie-Weiss law in the temperature range $200-375 \mathrm{~K}$ amounts to $2.6 \mu_{\mathrm{B}}$ (Fig. 2) and is close to the spin-only moment of $\mathrm{Ru}^{6+}$. Deviations of $\chi^{-1}(T)$ from linearity at approaching $T_{\mathrm{N}}$ may be attributed to weak frustration effects. 


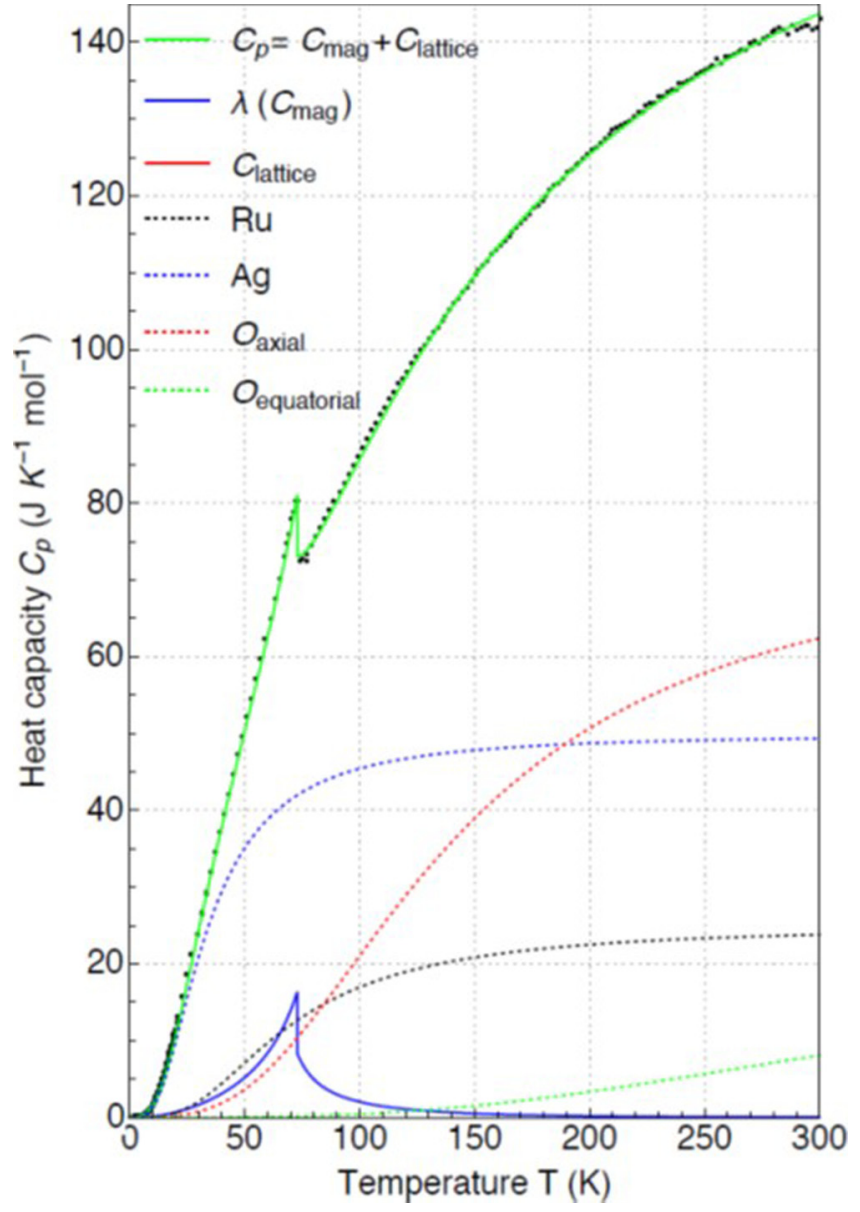

FIG. 3. Heat capacity $C_{\mathrm{p}}$ (experimental, black dots) fitted (green) by a lattice contribution $C_{\text {lattice }}$ as a sum (red) of four Debye functions (dotted) and a $\lambda$ function for the magnetic contribution $C_{\text {mag }}$ (blue).

Heat capacity measured as a function of temperature (Fig. 3) shows an anomaly at about $75 \mathrm{~K}$, which confirms the phase transition observed in the magnetic study. We fitted the heat capacity data applying a linear combination of four Debye functions with Debye temperatures of 138(2), 289(14), $582(5)$, and $1615(18) \mathrm{K}$, corresponding to the lattice contribution of the formula unit (two $\mathrm{Ag}$, one $\mathrm{Ru}$, three equatorial, and one axial $\mathrm{O}$ position) and an empiric lambda function [57]. The latter represents the magnetic contribution $C_{\mathrm{mag}}$ to the heat capacity $C_{\mathrm{p}}$ and is defined below and above the Néel temperature $T_{\text {Néel }}$ as follows, with the semiempirical parameters $K_{\mathrm{m}}, K_{\mathrm{n}}, m$, and $n$ as presented in Ref. [57]:

$$
\begin{aligned}
T & <T_{\text {Néel }}: 2 \mathrm{R} K_{m} \ln \left[\frac{1+\left(\frac{T}{T_{\text {Néel }}}\right)^{m}}{1-\left(\frac{T}{T_{\text {Néel }}}\right)^{m}}\right] \\
& \approx 2 \mathrm{R} K_{m}\left[\left(\frac{T}{T_{\text {Néel }}}\right)^{m}+\frac{1}{3}\left(\frac{T}{T_{\text {Néel }}}\right)^{3 m}+\frac{1}{5}\left(\frac{T}{T_{\text {Néel }}}\right)^{5 m}\right], \\
T & >T_{\text {Néel }}: 2 \mathrm{R} K_{n} \ln \left[\frac{\left(\frac{T}{T_{\text {Néel }}}\right)^{n}+1}{\left(\frac{T}{T_{\text {Néel }}}\right)^{n}-1}\right] \\
& \approx 2 \mathrm{R} K_{m}\left[\left(\frac{T}{T_{\text {Néel }}}\right)^{-n}+\frac{1}{3}\left(\frac{T}{T_{\text {Néel }}}\right)^{-3 n}+\frac{1}{5}\left(\frac{T}{T_{\text {Néel }}}\right)^{-5 n}\right] .
\end{aligned}
$$

A reasonable fit has been obtained with $K_{\mathrm{m}}=0.65(27)$, $K_{\mathrm{n}}=0.32(18), \quad m=2.1(4), \quad n=3.2(1.3), \quad$ and $T_{\text {Néel }}=$ 73(7) K. In both cases, the integration over $T$ of this supposed $\lambda$-shaped heat capacity anomaly around the Néel temperature, $C_{\text {mag, }, \lambda} / T$, delivers a value for the magnetic entropy of $S_{\text {mag }} \approx 8.4 \mathrm{~J} \mathrm{~K}^{-1} \mathrm{~mol}^{-1}$ instead of an expected value of $S_{\mathrm{mag}}=R \ln (2 S+1) \approx 9.1 \mathrm{~J} \mathrm{~K}^{-1} \mathrm{~mol}^{-1}$. Yet, regarding the empiric character of the fit and the lack of a model fitting a non-negligible shoulder in the heat capacity, once the lattice contribution is subtracted, at $\approx 15 \mathrm{~K}$, the value is sufficiently close to the theoretical one, to confirm the model, notably $S=1$.

The electrical resistivity (Fig. S3 [56]) measured as a function of temperature confirms the semiconducting nature of the compound. The low-temperature part of conductivity can be fitted by a variable range hopping model $\sigma=$ $\sigma_{0} \exp \left[-\left(T_{0} / T\right)^{\beta}\right]$ with $\beta=1 / 4$, and a Mott temperature of $T_{0} \approx 30 \mathrm{~K}$.

\section{B. Crystal structure}

The crystal structure of $\mathrm{Ag}_{2} \mathrm{RuO}_{4}$ was solved and refined using neutron and $\mathrm{x}$-ray powder data. The crystallographic details of the structure determination, the atomic parameters, and the relevant distances and angles are given in Tables I, S1 [56], and II, respectively. According to the quite consistent results of the set of structure refinements performed, ruthenium is in a fivefold, trigonal bipyramidal coordination by oxygen. The apical oxygen atoms of the bipyramids connect to form infinite chains $\mathrm{RuO}_{3 / 1} \mathrm{O}_{2 / 2}$ along the $a$ direction (Fig. 4).

The Ru-O bond distances vary from 1.752 to $1.776 \AA$ for equatorial and from 1.984 to $2.007 \AA$ for apical oxygen atoms, respectively. The $\mathrm{O}-\mathrm{Ru}-\mathrm{O}$ angles along the chain directions are nearly linear $\left(179.72^{\circ}\right)$, while the bipyramids are appreciably tilted with respect to neighboring polyhedra, as is indicated by a Ru-O-Ru angle of $125.64^{\circ}$. As illustrated by Figs. 4 and 5, the silver atoms are arranged to form approximately hexagonal tubes aligned parallel along [100] establishing a honeycomblike pattern. The tubes are centered by the $\mathrm{Ru} / \mathrm{O}$ chains, which thus form an almost ideal hexagonal rod packing. The individual rods are linked by silver atoms which are coordinated by five oxygen atoms, with the Ag-O bond distances ranging from 2.356 to $2.475 \AA$ (Fig. 5). Further, Figs. S4 [56], 4, and 5 display corresponding details of the crystal structures of $\mathrm{Ag}_{2} \mathrm{RuO}_{4}$ and $\mathrm{Na}_{2} \mathrm{RuO}_{4}$ for comparison, revealing a close relationship of the two structures.

\section{Magnetic ordering}

Upon cooling the sample below $T_{\mathrm{N}} \sim 75.4 \mathrm{~K}$, the appearance of new reflections in the neutron-diffraction patterns indicates the onset of long-range magnetic ordering of the $\mathrm{Ru}^{6+}$ ions. Figure S5 [56] shows the diffraction patterns of $\mathrm{Ag}_{2} \mathrm{RuO}_{4}$ collected with the diffractometer D20 at two different temperatures: $T=76 \mathrm{~K}$ (just above the magnetic transition temperature, lower curve) and $T=1.8 \mathrm{~K}$ (saturated magnetic state). The total Bragg intensity at $T=76 \mathrm{~K}$ is originating from nuclear scattering by the atoms of the chemical cell of $\mathrm{Ag}_{2} \mathrm{RuO}_{4}$. The additional reflections visible at $T=1.8 \mathrm{~K}$, on the other hand, are of magnetic origin. 
TABLE I. Crystallographic data and refinement details from PXRD and neutron powder diffraction (NPD).

\begin{tabular}{|c|c|c|c|c|}
\hline Compound & $\mathrm{Ag}_{2} \mathrm{RuO}_{4}$ & & & \\
\hline Diffraction mode & PXRD & NPD & NPD & NPD \\
\hline Wavelength & $1.5406 \AA$ & $1.4773 \AA$ & $1.4773 \AA$ & $1.4773 \AA$ \\
\hline Temperature & $298 \mathrm{~K}$ & $296 \mathrm{~K}$ & $76 \mathrm{~K}$ & $1.8 \mathrm{~K}$ \\
\hline Formula weight & 380.80 & & & \\
\hline Space group (no.) & Pnma (62) & & & \\
\hline \multicolumn{5}{|l|}{ Lattice constants } \\
\hline$a(\AA)$ & $7.1020(1)$ & $7.1018(3)$ & $7.0827(3)$ & $7.0855(2)$ \\
\hline$b(\AA)$ & $9.3370(2)$ & $9.3363(4)$ & $9.2992(4)$ & $9.2987(3)$ \\
\hline$c(\AA)$ & $5.5353(1)$ & $5.5355(2)$ & $5.5164(2)$ & $5.5150(2)$ \\
\hline$V\left(\AA^{3}\right)$ & $367.05(1)$ & $367.03(3)$ & $363.33(2)$ & $363.36(2)$ \\
\hline Rp / Rwp & $0.0293 / 0.0467$ & $0.047 / 0.049$ & $0.039 / 0.044$ & $0.040 / 0.045$ \\
\hline Goodness of fit & 2.36 & 2.79 & 3.07 & 3.38 \\
\hline
\end{tabular}

All additional lines originating from the neutron diffraction on the system of ordered $\mathrm{Ru}^{6+}$ magnetic moments can be indexed with integer indices within the parent unit cell of the crystal structure of $\mathrm{Ag}_{2} \mathrm{RuO}_{4}$. This implies a propagation vector of the magnetic structure of $\kappa=(000)$. The confirmation of the propagation vector was performed using the search routine K-SEARCH which is part of the FULLPROF suite [58]. This propagation vector, together with the known crystal symmetry of the chemical cell, and the positions of the $\mathrm{Ru}^{6+}$ magnetic ions, serve as an input for the symmetry analysis of the possible magnetic ordering schemes. The representation analysis of the low-temperature magnetic structure was carried out with the Program BASIREPS (part of the FULLPROF suite). All predicted symmetry-allowed spin configurations have been checked with Rietveld refinements by FULLPROF [59]. Very clearly, only one out of the eight irreducible representations ( $\Gamma 6$ for the $\mathrm{Ru}^{6+}$ ions in the notation of BASIREPS), which has two basis vectors, (100) and (001), provides sets of calculated diffraction peaks consistent with our experimental data. The corresponding magnetic space group (Shubnikov group) is Pnma. In this structure, the $\mathrm{Ru}^{6+}$ ions are coupled ferromagnetically along the chain direction [100], concerning the main component to the magnetic moment vector, pointing in direction [001], and antiferromagnetically concerning the minor component in the chain direction [100]. The chains are coupled antiferromagnetically (concerning the main component, ferromagnetically concerning the minor component) to the four closest chains in the $b-c$ plane and totally ferromagnetically to the two, by $3 \%$ farther chains in direction [001]. Out of the other irreducible representations, six do not explain the presence of the strong (010) and another one predicts intensity where none is observed. All possibilities of purely ferromagnetically ordered chains have been checked and could be excluded. One should note here that the chains are arranged pseudohexagonally. From Fig. S9 [56] one can state another important difference of the two farther neighbor chains in [001] as compared to the four closer ones: The two farther chains have the same orientation, whereas the four closer ones are "undulated" differently. As visible from Fig. S6 [56], the orientation of vectors with respect to the chains is different in $\mathrm{Na}_{2} \mathrm{RuO}_{4}$, where all chains are ferromagnetically arranged to each other, while the intrachain coupling is AFM.

The magnetic structures described by the two combinations (" \pm \pm " and " $\pm \mp "$-referring to the signs of the moment vector of the first Ru position in Table III) of the two basis vectors differ only in the direction of the magnetic moment with respect to the oxygen coordination of $\mathrm{Ru}^{6+}$ in the

TABLE II. Bond lengths $(\AA)$ and angles $\left(^{\circ}\right)$ (including those for $\mathrm{Na}_{2} \mathrm{RuO}_{4}$ from NPD at $293 \mathrm{~K}$ for comparison [44]).

\begin{tabular}{|c|c|c|c|c|c|}
\hline $\mathrm{M}-\mathrm{O}\left(\mathrm{Ag}_{2} \mathrm{RuO}_{4}\right)$ & $\mathrm{X}$ ray & NPD (296 K) & NPD $(1.8 \mathrm{~K})$ & $\mathrm{Na}_{2} \mathrm{RuO}_{4}(\mathrm{Ru} 1)$ & NPD (Ru2) \\
\hline $\mathrm{Ru}-\mathrm{O} 2$ & $1.752(9)$ & $1.755(2)$ & $1.764(2)$ & $1.77(1)$ & $1.782(7)$ \\
\hline $\mathrm{O} 3(\times 2)$ & $1.776(9)$ & $1.776(1)$ & $1.782(1)$ & $1.78(1) / 1.80(1)$ & $1.77(1) / 1.79(1)$ \\
\hline $\mathrm{O} 1$ & $1.984(4)$ & $1.995(2)$ & $1.990(2)$ & $1.975(7)$ & $1.997(6)$ \\
\hline $\mathrm{O} 1$ & $2.007(4)$ & $2.003(2)$ & $2.003(2)$ & $1.982(7)$ & $1.998(6)$ \\
\hline Ag- O1 & $2.356(6)$ & $2.349(1)$ & $2.342(1)$ & & \\
\hline $\mathrm{O} 3$ & $2.439(7)$ & $2.452(1)$ & $2.430(1)$ & & \\
\hline $\mathrm{O} 3$ & $2.456(6)$ & $2.455(1)$ & $2.434(1)$ & & \\
\hline $\mathrm{O} 3$ & $2.460(7)$ & $2.456(1)$ & $2.440(1)$ & & \\
\hline $\mathrm{O} 2$ & $2.475(7)$ & $2.471(1)$ & $2.460(1)$ & & \\
\hline Angles & $\mathrm{X}$ ray & NPD (296 K) & NPD $(1.8 \mathrm{~K})$ & $\mathrm{Na}_{2} \mathrm{RuO}_{4}(\mathrm{Ru} 1 / \mathrm{O} 7)$ & $\mathrm{Na}_{2} \mathrm{RuO}_{4}(\mathrm{Ru} 2 / \mathrm{O} 8)$ \\
\hline O1-Ru-O1 & $179.72(5)$ & $179.9(1)$ & $180.0(1)$ & $179.3(4)$ & $178.9(4)$ \\
\hline $\mathrm{Ru}-\mathrm{O} 1-\mathrm{Ru}$ & $125.64(4)$ & $125.3(1)$ & $125.06(9)$ & $124.4(5)$ & $123.3(4)$ \\
\hline
\end{tabular}



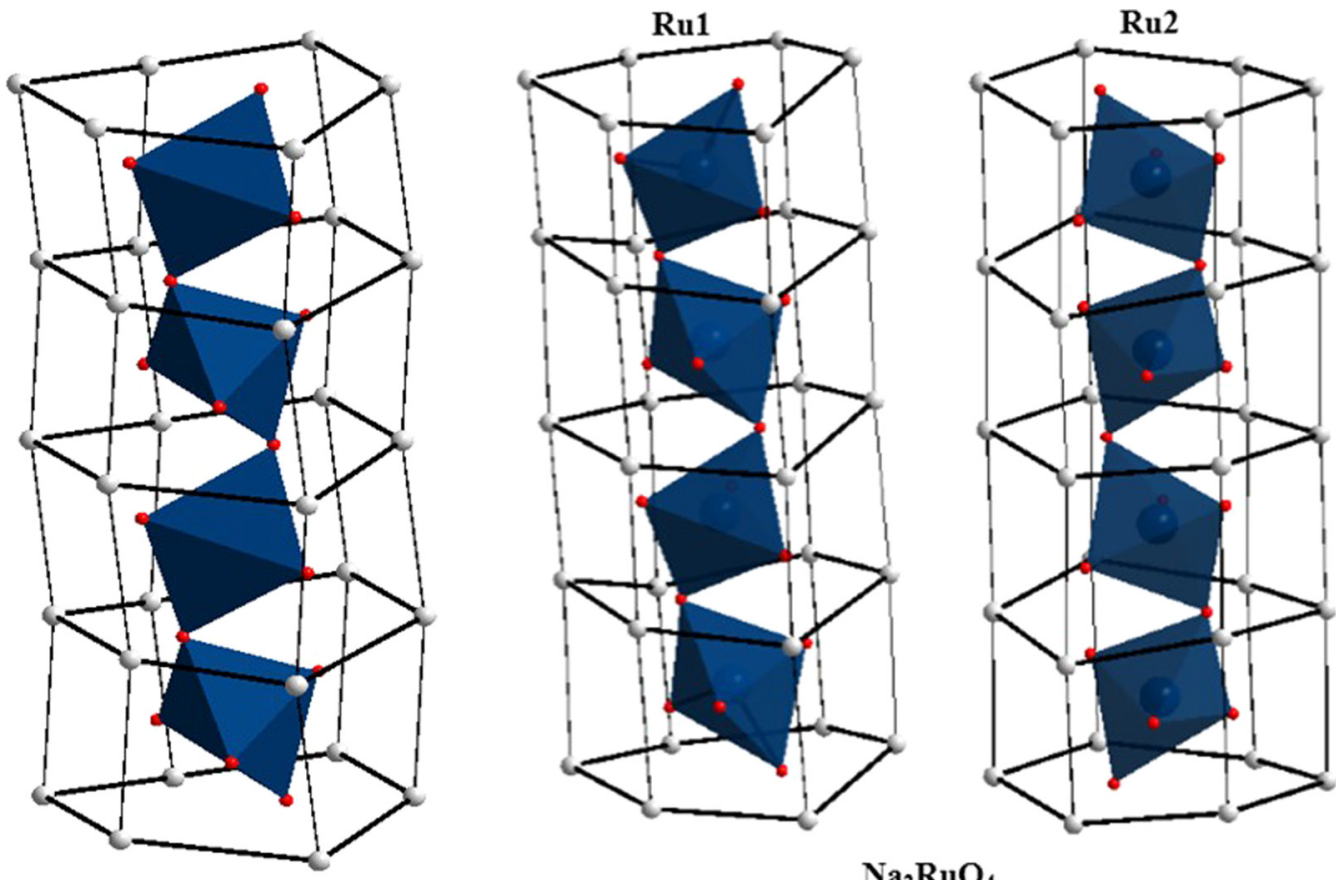

\section{$\mathrm{Na}_{2} \mathrm{RuO}_{4}$}

FIG. 4. Visualization of individual $\left(\mathrm{RuO}_{5}\right)$ chains and the surrounding monovalent countercations for $\mathrm{Ag}_{2} \mathrm{RuO}_{4}$ (left) and for $\mathrm{Na}_{2} \mathrm{RuO}_{4}$ (right), where the two columns correspond to the independent Ru1 (left) and Ru2 (right) sites. $\mathrm{RuO}_{5}$ polyhedra: blue, oxygen; red spheres, silver; gray spheres, sodium.

chemical cell, pointing in the trigonal base plane of the trigonal bipyramids or out of it, respectively. Precise checking of these two possibilities has shown that the difference is nonsignificant in any diffraction experiment, as $\mathrm{Ru}^{6+}$ occupies nearly (within $3 \sigma$ ) the ideal position $\left(1 / 41 / 4 \frac{1}{4}\right)$, where a shift of the magnetic unit cell by $\left[\begin{array}{lll}1 / 2 & 0 & 0\end{array}\right]$ becomes possible without altering diffraction intensities at all. However, in $\mathrm{Na}_{2} \mathrm{RuO}_{4}$, the vectors lay inside the plane, as Fig. S6 [56] shows; by analogy, one can conclude that this orientation is also the most likely one in the case of $\mathrm{Ag}_{2} \mathrm{RuO}_{4}$. The refined components of magnetic moments along $a$ and $c$ stay roughly in the same proportion at all temperatures. The magnetic moment lies in the $a-c$ plane and is aligned predominantly along $c$ with a weaker $a$ component. At $1.8 \mathrm{~K}$, the two components are refined to $M_{c}=1.45(2) \mu_{\mathrm{B}}$ and $M_{a}=0.76(1) \mu_{\mathrm{B}}$; in spherical coordinates, this corresponds to a magnetic moment of $M=1.547(7) \mu_{\mathrm{B}}$ at an angle $\Theta=29.7(4)^{\circ}$ from the $c$ axis. An illustration of the two possible magnetic structure models for $\mathrm{Ag}_{2} \mathrm{RuO}_{4}$ is shown in Fig. 6. There is only one irreducible representation fitting the diffraction data, and the absolute amount of the two basis vectors it contains converges to reproducible values of $M_{\mathrm{a}}$ and $M_{\mathrm{c}}$. Yet, the signs of the two basis vectors relative to each other are interchangeable. Due to the pseudosymmetry described above, the two slightly
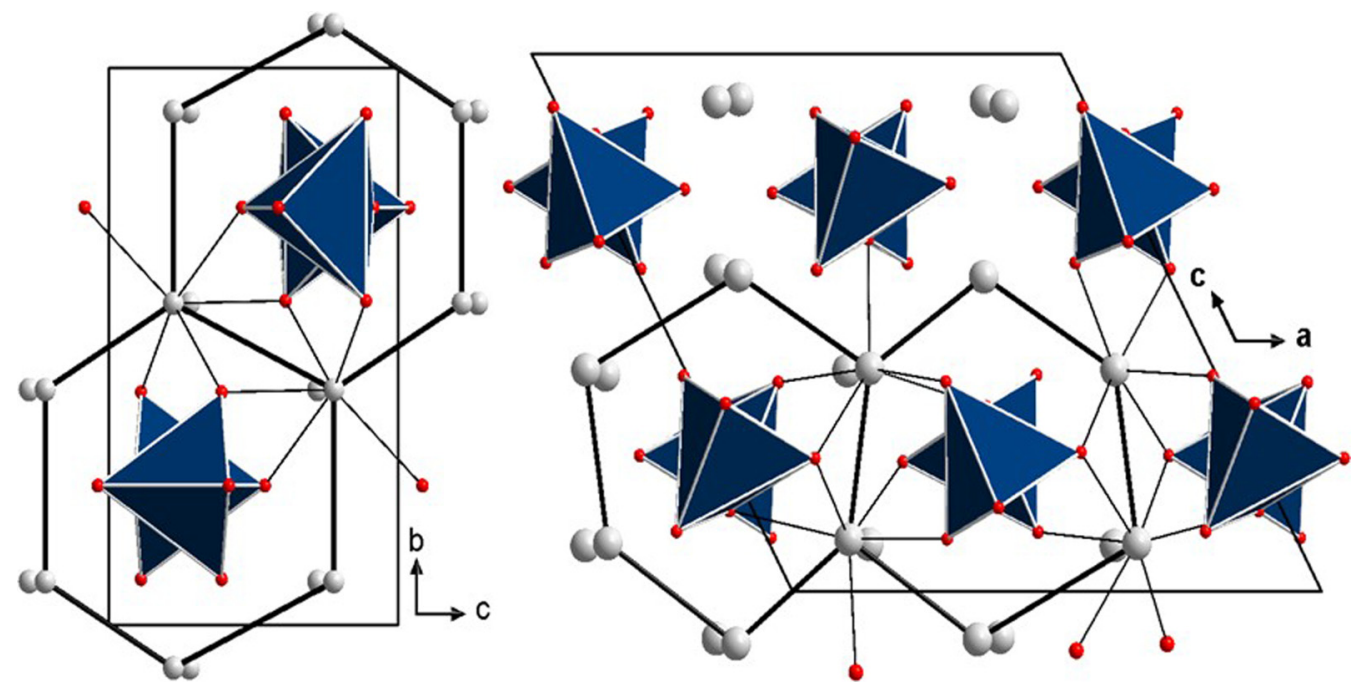

FIG. 5. Connectivity between $\left(\mathrm{RuO}_{5}\right)$ chains in $\mathrm{Ag}_{2} \mathrm{RuO}_{4}$ (left) and $\mathrm{Na}_{2} \mathrm{RuO}_{4}$ (right) as mediated by $\mathrm{Na}^{+}$and $\mathrm{Ag}^{+}$, respectively. Thin black lines cover distance ranges from 2.356 to $2.477 \AA$ A. Same color code as in Fig. 4. 
TABLE III. Magnetic moment vectors for the four equivalent Ru positions $\left(x \approx-0.2497, z \approx-0.2506, M_{\mathrm{a}} \approx 0.76 \mu_{\mathrm{B}}, M_{\mathrm{c}} \approx 1.45 \mu_{\mathrm{B}}\right.$, $M \approx 1.55 \mu_{\mathrm{B}}, \Theta \approx 30$ ).

\begin{tabular}{|c|c|c|c|c|c|c|c|}
\hline \multirow{2}{*}{$\frac{\text { Ion }}{\text { Ru1 }}$} & \multicolumn{3}{|c|}{ Fractional coordinates } & \multicolumn{2}{|c|}{ Cartesian vector } & \multicolumn{2}{|c|}{ Polar vector } \\
\hline & $+x$ & $3 / 4$ & $+z$ & $+M_{\mathrm{a}}$ & $\pm M_{\mathrm{c}}$ & $+M$ & $\pm \Theta$ \\
\hline $\mathrm{Ru} 2$ & $-x+1 / 2$ & $1 / 4$ & $+z+1 / 2$ & $+M_{\mathrm{a}}$ & $\mp M_{\mathrm{c}}$ & $+M$ & $\mp \Theta+180^{\circ}$ \\
\hline Ru3 & $-x$ & $1 / 4$ & $-z$ & $-M_{\mathrm{a}}$ & $\mp M_{\mathrm{c}}$ & $+M$ & $\pm \Theta+180^{\circ}$ \\
\hline $\mathrm{Ru} 4$ & $+x+1 / 2$ & $3 / 4$ & $-z+1 / 2$ & $-M_{\mathrm{a}}$ & $\pm M_{\mathrm{c}}$ & $+M$ & $\mp \Theta$ \\
\hline
\end{tabular}

different magnetic structures are virtually undistinguishable and can (nearly) be transformed into each other by a simple translation. The chemical environment, however, is different for both solutions; however, in the diffraction experiment, there is no observable interference between the nuclear and the magnetic diffraction.

An additional difficulty in refining the exact value of the magnetic moment arises from the unknown magnetic form factor of thermal neutron scattering for the $\mathrm{Ru}^{6+}$ ions $\left(4 d^{2}\right.$ electronic configurations). To our knowledge, it has never been measured, or calculated to date. All modern Rietveld refinement codes use the approximations for the magnetic form factors as tabulated in Ref. [60], which only contains form factors for the atom $\mathrm{Ru}$ and the cation $\mathrm{Ru}^{+}$, but not for higher oxidation states. Obviously, these approximations cannot be used for the $\mathrm{Ru}^{6+}$ ion, since its outer electronic shells are much more strongly localized. If one tries the refinement with either of the two form factors, immediately an artificial unphysical "overall thermal parameter" for the magnetic phase needs to be introduced in order to obtain a reasonable fit quality. The exact determination of the $\mathrm{Ru}^{6+}$ form factor is beyond the scope of this investigation, therefore the same approach as in Ref. [45] was used: The magnetic form factors of various lighter neighboring $4 d$ elements in different oxidation states have been used in the refinement, and the best profile description (without necessity for any artificial overall thermal parameter for the magnetic phase) has been retained. The ion $\mathrm{Zr}^{+}$turned out to be the best candidate, in contrast to Ref. [45], where the Y atom form factor had been chosen. With the assumption of a slight preferred orientation of particles adopting the pseudohexagonal habit (needles or platelets) and a correction after March, the $\mathrm{Y}$ atom gives a better fit, but only with a considerable-and unlikely-texture correction (needle habit), and an unexpectedly high magnetic moment. Hence, we have used the known and tabulated approximation for the $\mathrm{Zr}^{+}$magnetic form factor in our refinements. Thanks to our high counting statistics in the difference pattern, the differences between different candidates in the Rietveld fit are significant, which gives us confidence that the approximation by $\mathrm{Zr}^{+}$can be considered as the closest possible and therefore delivers best possible absolute values for the magnetic moment.

In order to estimate the possible uncertainty in the determination of the absolute value of the $\mathrm{Ru}^{6+}$ magnetic moment, it is worth comparing the refined values for different trial magnetic ions. Without preferred orientation (the refinement
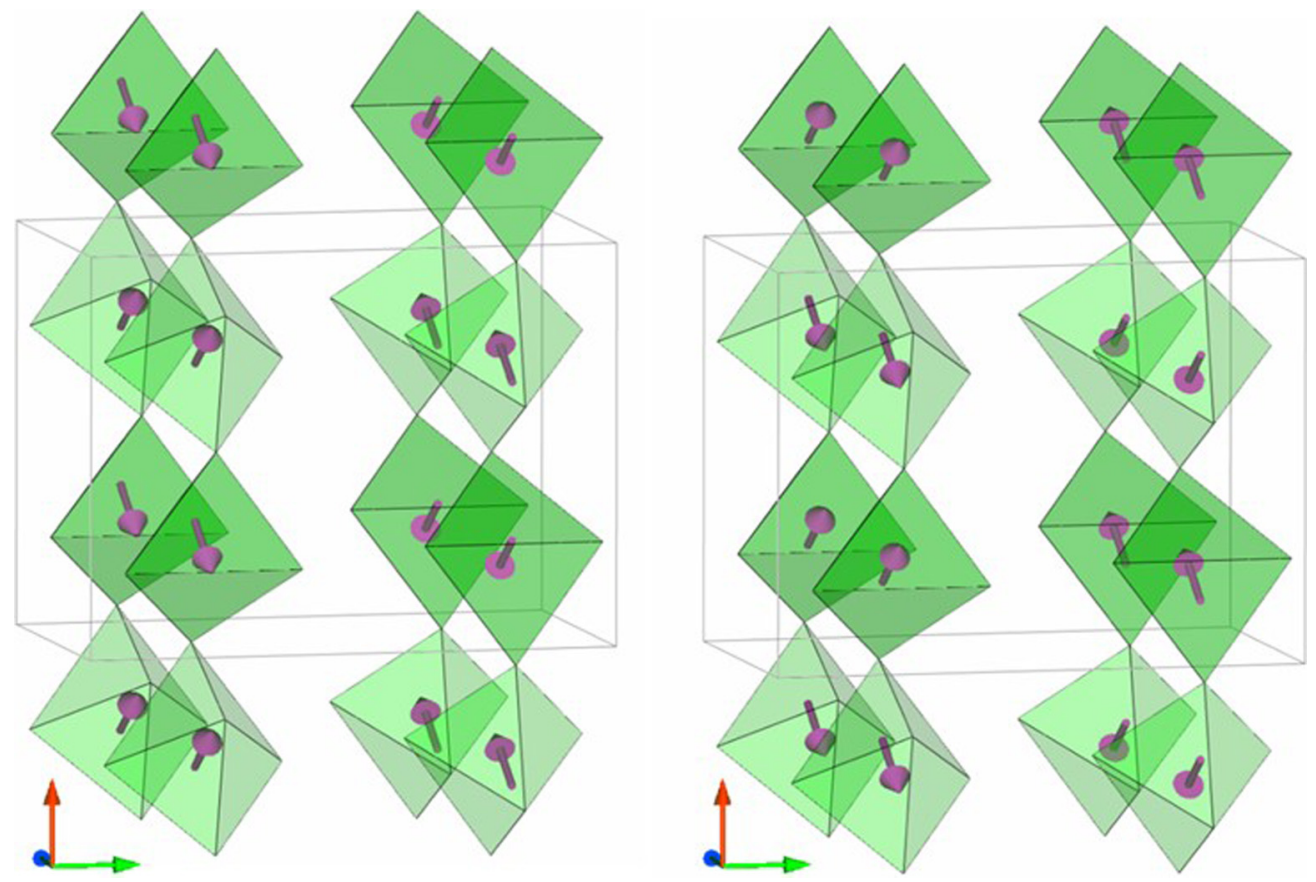

FIG. 6. Projection of the two possible magnetic structures of $\mathrm{Ag}_{2} \mathrm{RuO}_{4}$ along [001], the $a$ axis (direction of the chains [100]) pointing upwards in the paper plane. Only the unit-cell boundary, the $\left[\mathrm{RuO}_{5}\right]$ coordination bipyramids around $\mathrm{Ru}^{6+}$ (green), and the magnetic moment vectors (purple) are represented. Crystallographic axes are indicated in red $(a)$, green $(b)$, and blue $(c)$. 


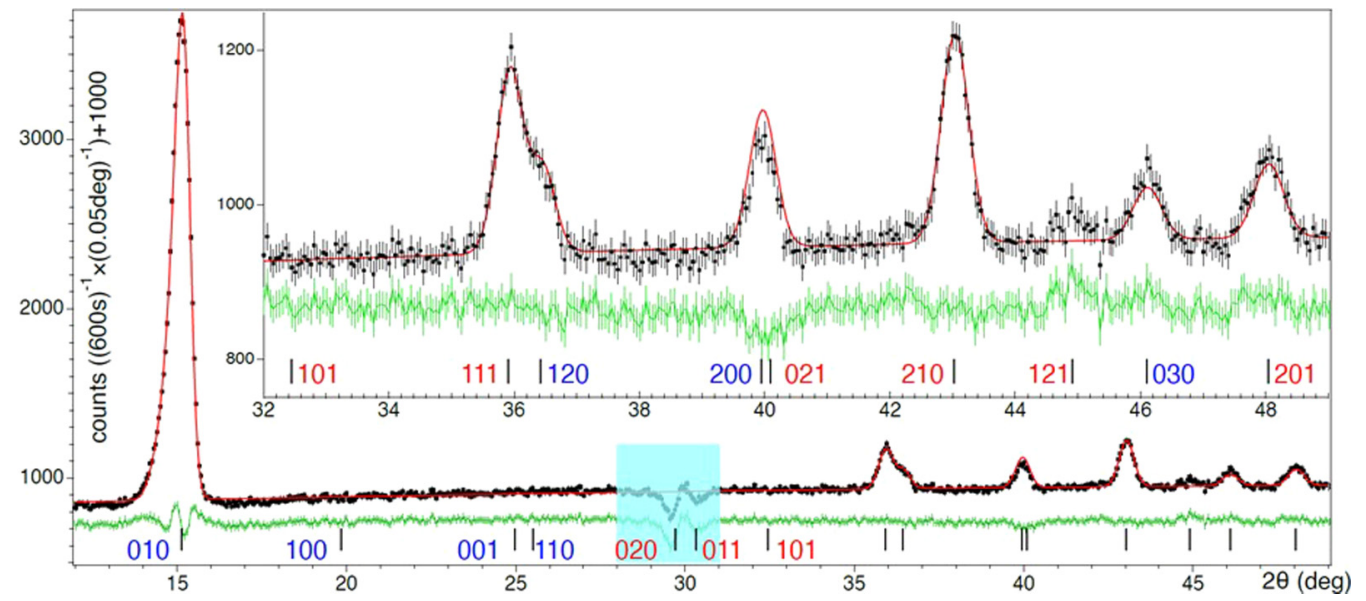

FIG. 7. Rietveld fit of the difference pattern (pattern at $1.8 \mathrm{~K}$ minus isotropic thermal expansion-corrected pattern at $76 \mathrm{~K}$, black dots-an offset of 1000 counts has been applied) with the pure magnetic phase. Positions of strong nuclear peaks are excluded because of a remaining misfit due to thermal expansion. Black lines indicate Bragg peak positions; the corresponding indexing is given in blue for pure magnetic peaks, and in red for nuclear peaks with potential magnetic scattering contribution. The peaks (111), (021), (210), and (201) are at the position of a stronger nuclear peak, whereas one observes no magnetic scattering intensity at the peak positions (020), (011), (101), and (121). The latter seems to be of nonzero magnetic scattering intensity in the difference pattern for reasons of counting statistics (the nuclear scattering intensity is about three times as high as the magnetic peak (210)), thermal expansion, and the Debye-Waller factor.

of the full pattern, including magnetic and nuclear structure, gives no hint for a significant deviation from random orientation), they range from $1.455(6) \mu_{\mathrm{B}}$ for $\mathrm{Mo}^{+}\left(\chi^{2}=\right.$ 2.28), 1.494(6) $\mu_{\mathrm{B}}$ for $\mathrm{Nb}^{+}\left(\chi^{2}=2.05\right)$, and 1.547(7) $\mu_{\mathrm{B}}$ for $\mathrm{Zr}^{+}\left(\chi^{2}=2.01\right)$ to $1.635(6) \mu_{\mathrm{B}}$ for $\mathrm{Y}\left(\chi^{2}=2.25\right)$. While (without the assumption of texture) $\mathrm{Mo}^{+}$and $\mathrm{Y}$ can be clearly excluded due to lack of fitting quality, the discrepancy of the values obtained with $\mathrm{Nb}^{+}$and $\mathrm{Zr}^{+}$gives an estimate of the uncertainty of the absolute value of the magnetic moment of less than $\pm 5 \%$. Thus, we shall retain the value for $\mathrm{Zr}^{+}$in the following.

\section{Temperature dependence and magnetostriction}

The powder-diffraction refinement on the difference pattern is shown in Fig. 7. From respective refinements at various temperatures, the temperature dependence of the ordered magnetic $\mathrm{Ru}^{6+}$ moment magnitude has been extracted; it is presented in Fig. 8. In order to estimate the AFM transition temperature, the data of $M_{\mathrm{Ru}}(T)$ shown in Fig. 8 were fitted to an empirical formula (1):

$$
M_{\mathrm{Ru}}(T)=M_{0}\left[1-\left(T / T_{\mathrm{N}}\right)^{\alpha}\right]^{\beta} .
$$

The four free parameters refine to a saturated magnetic moment at $T=0$ of $M_{0}=1.53(1) \mu_{\mathrm{B}}$, a Néel temperature of $T_{\mathrm{N}}=75.8(4)$, and the exponents $\alpha=3.3(5)$ and $\beta=$ $0.35(6)$. The inclination of the moment vector with respect to the $c$ axis stays constant over temperature with $\Theta=30.5(4)^{\circ}$, in agreement with $M=1.547(7) \mu_{\mathrm{B}}$ and $\Theta=29.7(4)$ obtained from the refinement of the difference pattern at base temperature.

It is worth investigating potential magnetoelastic effects. Indeed, there is a clear discontinuity of lattice constants and unit-cell volume below the Néel temperature. One can fit the lattice constants above $T_{\mathrm{N}}$ to a modified Einstein function:

$$
x(T)=x_{0}+\left(E_{0}+E_{1} x+E_{2} x^{2}\right) /\left[\exp \left(\theta_{E} / T\right)-1\right] .
$$

Below $T_{\mathrm{N}}$ one observes a strong negative deviation from the Einstein fit, for the lattice constants $b$ and $c$ and the unitcell volume $V$, whereas the effect is insignificantly positive on the lattice constant $a$, as visible in Fig. S7 [56].

The unit-cell volume has been modeled in addition by a linear combination of four Debye functions with Debye temperatures of $138,289,582$, and $1615 \mathrm{~K}$, in analogy to and as obtained from the fit of the heat capacity data (Fig. 3) considering a unique proportionality parameter $\gamma / B_{0}$ (Grüneisen/bulk modulus) for all terms [61]. The result is shown (orange) along with the Einstein model fit (black) in Fig. S7 [56]. As expected, the two models diverge at lower temperature. The strain $\Delta V / V$ (Fig. S8 [56]) is proportional to the squared magnetic moment, the order parameter, and reaches a value of $480 \times 10^{-6}$ in the Einstein case and $320 \times$ $10^{-6}$ in the Debye case, which are values commonly observed for similar magnetic moments [61].

\section{E. Magnetic susceptibility}

The discontinuity of the heat capacity at $\approx 75 \mathrm{~K}$ is in agreement with the AFM transition temperature of 75.5(8) K as obtained from the neutron-diffraction experiments. Also, the magnetic susceptibility measurement indicates AFM coupling below $75.4 \mathrm{~K}$. A dominant AFM exchange interaction is evident from the magnetic susceptibility data. The broad maximum (disrupted below $T_{\mathrm{N}}$ ) is characteristic for shortrange AFM ordering in low-dimensional systems, i.e., an AFM coupled chain system. Above $200 \mathrm{~K}$, the susceptibility can be modeled by a Curie-Weiss law with a Weiss constant of $\theta \approx 155(1) \mathrm{K}(35 \mathrm{Oe})$ or $155.9(8) \mathrm{K}(70 \mathrm{Oe})$ and a Curie constant of $C \approx 0.860(2)$, respectively $0.875(2) \mathrm{cm}^{3} \mathrm{~K} \mathrm{~mol}^{-1}$, corresponding to an effective moment of $\mu_{\text {eff }} \approx 2.623(3)$, respectively $2.646(3) \mu_{\mathrm{B}}$. The value of the Curie constant close to $1 \mathrm{~cm}^{3} \mathrm{~K} \mathrm{~mol}^{-1}$ is $g$ expected for a spin-only contribution from the $d^{2}$ ion $\mathrm{Ru}^{6+}$ with a Landé factor of $g \approx 1.855(2)$, 

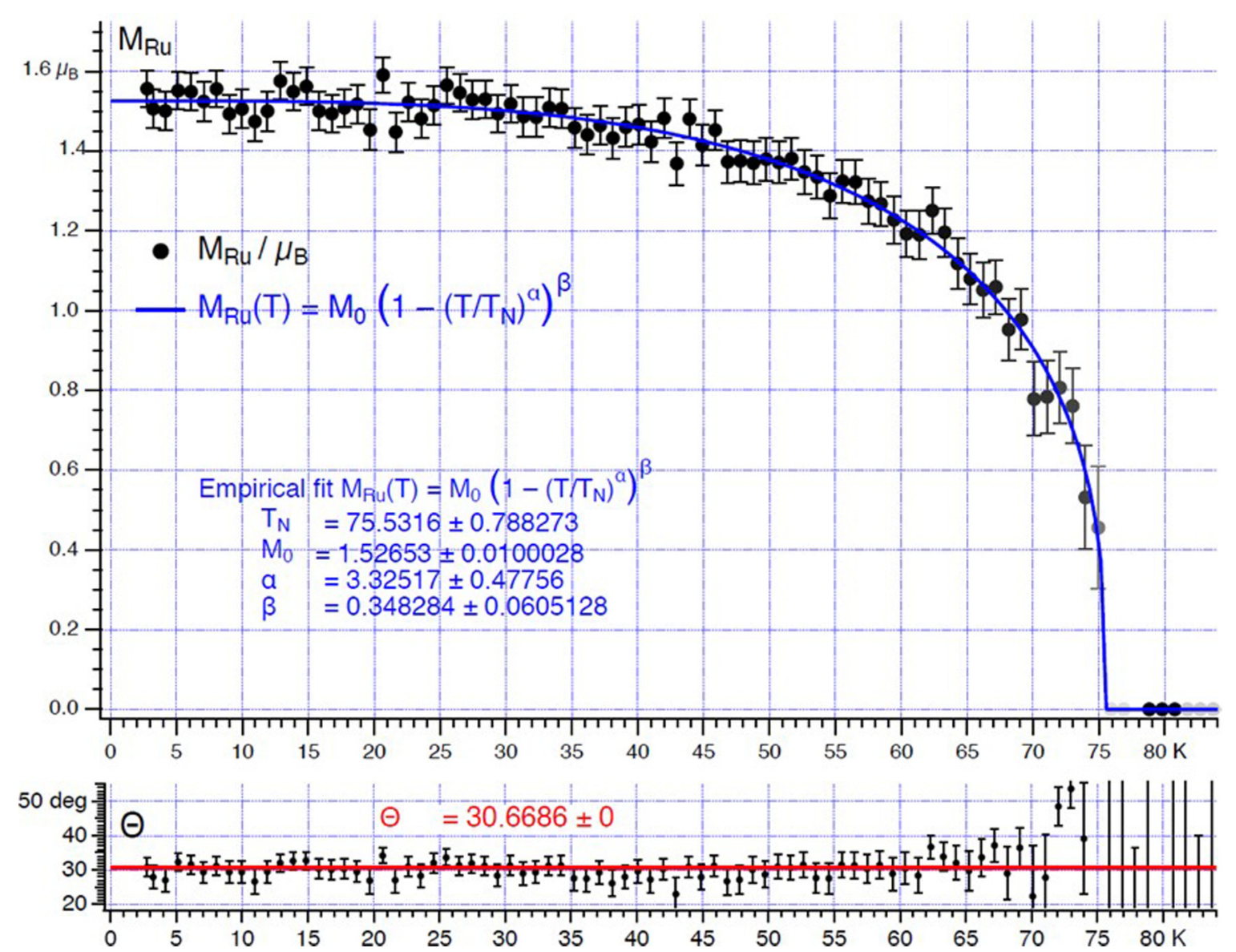

FIG. 8. Temperature dependence of the ordered $\mathrm{Ru}^{6+}$ magnetic moment magnitude in $\mathrm{Ag}_{2} \mathrm{RuO}_{4}$. Circles correspond to the results of the sequential refinement of a thermodiffractometry. The solid blue line is a fit of the $M_{\mathrm{Ru}}(T)$ data with Eq. (1) in the text. Below is shown the angle of the moment with respect to the $c$ axis as a function of temperature.

respectively $1.871(2)$ and $S=1$ [45]. From the Weiss temperature $\Theta$ one can deduce a model-free first estimate of the nearest exchange coupling through $-J_{\|}=3 k_{\mathrm{B}} \Theta /[2 z S(S+$ 1)] with $z=2$ nearest neighbors and $\operatorname{spin} S=1$. One obtains $J_{\|}=-58.0(3) \mathrm{K}$ for $35 \mathrm{kOe}$, respectively $-58.5(3) \mathrm{K}$ for 70 kOe. One notes that the Weiss constant is lower than the one found for $\mathrm{Na}_{2} \mathrm{RuO}_{4}$, indicating a weaker AFM exchange interaction. The obtained smaller value of $g$ could be due to the effect of spin-orbit coupling and orbital contribution of the magnetic moment, as we discuss below.

Due to the considerable interchain interactions, resulting in long-range magnetic ordering, no attempt was made to fit an isolated Heisenberg chain model. In such a one, from $T\left(\chi_{\max }\right) \approx 76$ or $69 \mathrm{~K}$ (considering that, without long-range ordering, the maximum would lie at such a lower temperature) and $\chi_{\max } \approx 0.0033 \mathrm{emu} / \mathrm{mol}$, one would conclude, referring to Table 7.1 in Ref. [62], that $2 J_{\|} \approx-56$ or $-52 \mathrm{~K}$ and $g \approx 1.7$ or 1.6 , respectively.

Below $200 \mathrm{~K}$ and above the Néel temperature of $76 \mathrm{~K}$ we attempted a fit of the susceptibility with a classical infinite chain model $[63,64]$ in order to extract an estimate of the nearest-neighbor exchange parameter along the chains:

$$
\chi=N g^{2} \mu_{\mathrm{B}}^{2} S(S+1) / 3 k T(1+u) /(1-u),
$$

with $u=\operatorname{coth}\left[2 J_{\|} S(S+1) / k T\right]-k T /\left[2 J_{\|} S(S+1)\right]$.
The obtained exchange parameter of $2 J_{\|}=-74(1) \mathrm{K}$ (35 Oe), respectively $-73.81(8) \mathrm{K}$, and Landé factor $g=$ $1.7719(8)$, respectively $1.7854(6)$, are close to the values obtained for $\mathrm{Na}_{2} \mathrm{RuO}_{4}\left(2 J_{\|}=-86 \mathrm{~K}\right)$ [45]. The deviation of $g$ from 2 indicates presence of a low-level spin-orbit coupling but may as well be the result of the crude approximation of this $S=1$ system by a classical spin-chain model. To obtain an estimate of the strength of the interchain coupling parameter $\left|2 J_{\perp}\right|$ we use the following mean-field approximation [65]:

$$
\left|2 J_{\perp}\right|=T_{\mathrm{N}} /\left\{1.28 n\left[\ln \left(5.8\left|2 J_{\|}\right| / T_{\mathrm{N}}\right)\right] 1 / 2\right\} .
$$

With $n=6$ neighboring chains, we obtain $\left|2 J_{\perp}\right| \approx 7.5 \mathrm{~K}$. The sign of the interaction parameter cannot be evaluated, and as we deal with AFM and FM coupling between chains, as results from the neutron-diffraction study, we cannot elucidate it here. Also, we have to keep in mind that four neighboring chains are slightly closer (and couple mostly antiferromagnetically) and two are slightly further away (and couple exclusively ferromagnetically).

The ratio $2 J_{\perp} / 2 J_{\|} \approx 10 \%$ of inter- to intrachain exchange reflects the strong magnetic anisotropy in this material. The trigonal bipyramids coordinating ruthenium have a $D_{3 \mathrm{~h}}$ point symmetry and the axially elongated coordination results in singly occupied $d_{y z}$ and $d_{z x}$ orbitals (not degenerated due to the distortion of the trigonal bipyramids). The approximate $120^{\circ}$ angle between the $z$ axes of the orbital systems of two 


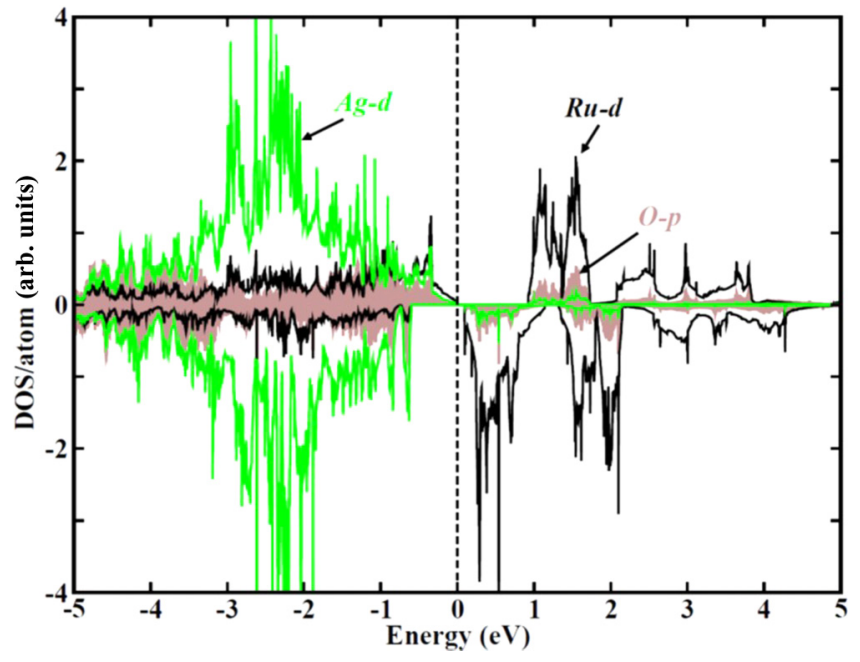

FIG. 9. GGA $+U$ with $U_{\text {eff }}=2 \mathrm{eV}$ spin-polarized density of states projected onto Ag- $4 d$ (light solid line), Ru-4d (dark line), and O-2 $p$ (filled) states. Two channels represent majority-spin (top) and minority-spin (bottom) channels. The Fermi level is marked at zero on the energy scale.

adjacent $\mathrm{Ru}^{6+}$ ions allows for sufficient overlap to produce an FM-AFM intrachain coupling by kinetic exchange.

\section{F. Band structure and density of states}

Figure 9 shows the electronic density of states (DOS) calculated within $\mathrm{GGA}+U\left(U_{\text {eff }}=2 \mathrm{eV}\right)$ for the FM state. The top and bottom panels show the majority- and minorityspin channels, respectively, while the Fermi energy is set at zero. Notably, even with inclusion of very small $U$ at the Ru site the electronic structure shows an insulating solution with a very small gap of $0.1 \mathrm{eV}$. The $\mathrm{Ag}-4 d$ states are completely filled in both spin channels, resulting in a nominal valance state of +1 for silver. The DOS shows that for Ru- $4 d$ states the majority-spin channel is partially filled and partially empty, whereas the minority-spin channel is completely empty. Due to the distorted nature of the trigonal bipyramidal $\left[\mathrm{RuO}_{5}\right]$ environment along with the tilting and rotation of the trigonal bipyramid along the apical direction, all $d$ states are highly mixed up. Interestingly, there is substantial hybridization between $\mathrm{O}-2 p$ states with $\mathrm{Ru}-4 d$ states, as indicated by the overlap region in the DOS. The calculated magnetic moment at the Ru site is $1.17(1.33) \mu_{\mathrm{B}} /$ site for $U_{\text {eff }}=2(4) \mathrm{eV}$, while the total magnetic moment is $2 \mu_{\mathrm{B}} / \mathrm{f}$.u.

We crosschecked the band-structure calculations and the calculated exchange interactions for the range of $U_{\text {eff }}$ from 1 to $4 \mathrm{eV}$. We found that for $U_{\text {eff }}=1 \mathrm{eV}$ the electronic structure gives a metallic state, while for $U_{\text {eff }}>1 \mathrm{eV}$ the band-structure calculation results in a gapped insulating state. We also found that for $U_{\text {eff }}=2$ to $4 \mathrm{eV}$, apart from the increase in the magnetic moment at the $\mathrm{Ru}$ site and band gap, no major changes occurred in the electronic structure. Although the absolute value of the exchange interactions changes due to a change of $U$, the main trend remains unchanged. We also compared with the literature regarding the $U$ value of the $\mathrm{Ru}$, and we found that it is commonly assumed to lie between
2 and $3.5 \mathrm{eV}[16,17,38,66-72]$. The combination of DOS and calculated magnetic moment suggested that $\mathrm{Ru}$ in this material is in the +6 nominal valance state with $4 d^{2}$ electronic configuration, with two states filled in the majority-spin channel and all remaining states in both spin channels being empty. Therefore, the underlying low-energy magnetic model of the present compound can be considered effectively as an $S=1$ spin system. To understand the ground-state magnetic behavior of the said material better, we performed the totalenergy investigation among different magnetic configurations of this effective $S=1$ spin system. The effect of strong hybridization is also reflected by a substantially large induced magnetic moment at the oxygen sites of $0.14(0.1) \mu_{\mathrm{B}}$ for $U_{\text {eff }}=2(4) \mathrm{eV}$.

The experiments show clear indication of competition of both FM and AFM exchange interactions between different $\mathrm{Ru}$ sites. However, microscopically the individual nature of each different type of exchange interactions is not clear. Therefore, we calculated the magnetic exchange interactions between different Ru sites of the said material through a DFT based first-principles approach. Magnetic exchange interactions can be found using the extended Kugel-Khomskii model [73-75], following the knowledge of the orbital-dependent hopping interactions, on-site Hubbard $U$, and charge-transfer energies between different electronic states and orbitals. However, such an approach would require precise evaluation of the complicated hopping integral and charge-transfer energy among the different orbitals connected through complex superexchange pathways involving different types of atoms, which is hardly feasible for such an intricate material. Alternatively, one can calculate magnetic exchange interactions via the total-energy calculations and map the DFT total energies for different types of spin arrangements to the corresponding Ising-like model $\left(E^{\text {total }}=\sum_{i j} J_{i j} S_{i}^{z} S_{j}^{z}\right)[75,76]$, where $S^{z}$ 's are the effective spin value at the site $i$ and $j$ and $J_{i j}$ is the exchange interaction between them. Although the total-energy calculation depends on the choice of Hubbard $U$, exchange correlation, etc., this method is able to provide the trends of exchange interactions reasonably well [75-79]. In the calculations, we consider the three most relevant isotropic exchange interaction ( $J$ 's) paths connecting different $\mathrm{Ru}$ sites. We considered intrachain superexchange interaction $\left(J_{1} ; \measuredangle \mathrm{Ru}-\mathrm{O}\right.$ $\mathrm{Ru}=125.6^{\circ}, \mathrm{Ru}-\mathrm{Ru}=3.11 \AA$ along the crystallographic $a$ direction), interchain $\left(J_{2} ; \mathrm{Ru}-\mathrm{Ru}=5.41 \AA\right)$ in the crystallographic $b$-c plane, and interchain $\left(J_{3} ; \mathrm{Ru}-\mathrm{Ru}=5.52 \AA\right)$ along the crystallographic $c$ direction, as illustrated in Fig. 10. The exchange interactions are calculated with $U_{\text {eff }}=2 \mathrm{eV}$. The total-energy calculations show the superexchange (Ru-O-Ru) intrachain $\left(J_{1}\right)$ interaction is strongest $(24.7 \mathrm{meV})$ and FM in nature, whereas the first neighbor interchain interaction $\left(J_{2}\right)$ is AFM with a moderate strength of $18.1 \mathrm{meV}$. The next-neighboring interchain interaction $\left(J_{3}\right)$ in the $b$-c plane is very weakly $(1.6 \mathrm{meV})$ ferromagnetic, compared to $J_{1}$ and $J_{2}$. Therefore, we can regard this compound to feature FM coupled chains running along the crystallographic $a$ direction, which are coupled both antiferromagnetically (along the $c$ direction) and ferromagnetically (in the diagonal $b c$ plane). To understand the effect of Coulomb $U$ on the calculated values of magnetic exchange interactions, we cross checked the $J$ values for GGA $+U_{\text {eff }}(=4 \mathrm{eV})$ and we found the trend to 


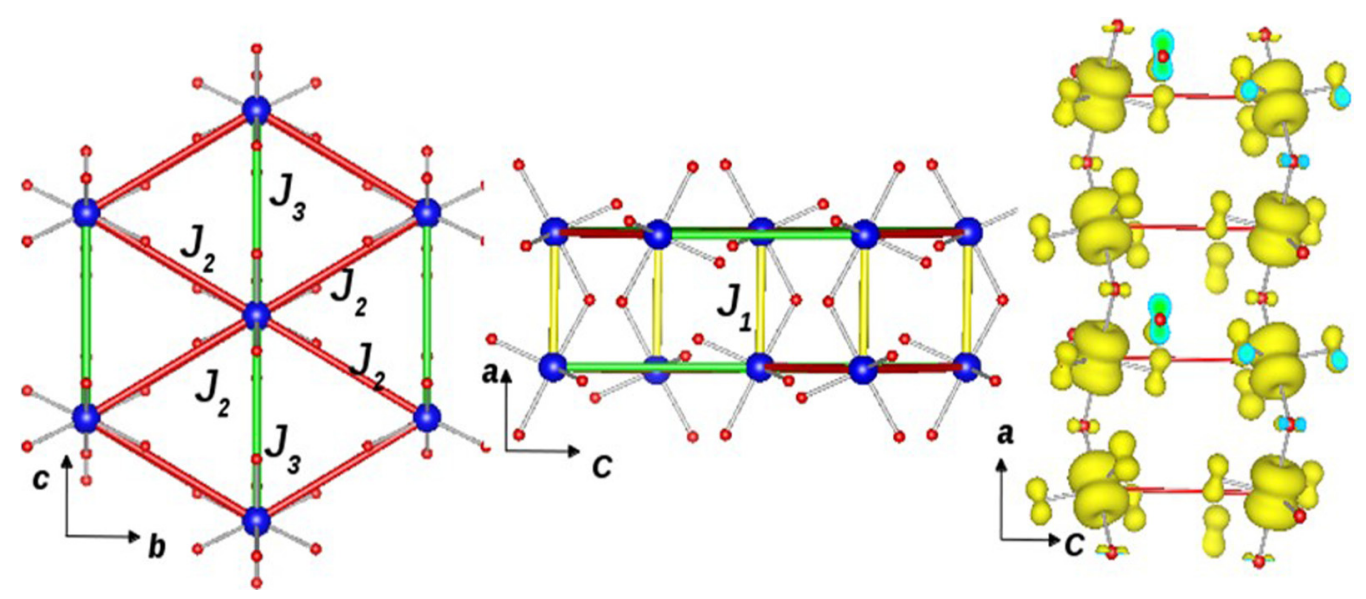

FIG. 10. The Ru only sublattice of $\mathrm{Ag}_{2} \mathrm{RuO}_{4}$. Shown are the dominant exchange interactions paths $J_{1}$ (intrachain), $J_{2}$, and $J_{3}$ (interchain) connecting between different $\mathrm{Ru}$ sites. The two figures on the left show two different view directions. Right panel: Three-dimensional magnetization density plot of $\mathrm{Ag}_{2} \mathrm{RuO}_{4}$ calculated through GGA $+U$. The isovalue was chosen to be $60 e^{-} / \AA^{3}$.

remain unaltered although the actual values of the exchange interactions $\left[J_{1}=20.4 \mathrm{meV}(\mathrm{FM}), J_{2}=11.1 \mathrm{meV}\right.$ (AFM), $\left.J_{3}=1.2 \mathrm{meV}(\mathrm{FM})\right]$ are changing.

The nature of the FM $J_{1}$ and AFM $J_{2}$ can be qualitatively understood from the Goodenough-Kanamori rules [80,81]. The superexchange angle connecting two Ru sites in the $J_{1}$ interaction path $\measuredangle \mathrm{Ru}-\mathrm{O}-\mathrm{Ru}=125.64$ is far away from ideal $180^{\circ}$. Therefore, the FM component of the $J_{1}$ dominates over the AFM counterpart and finally stabilizes the ferromagnetic nature, whereas the in-plane canting of $\mathrm{Ru}$ spins induces the AFM nature for the $J_{2}$ interchain interaction.

To understand the orbital contribution to magnetic moment leading to a reduction of the $g$ value, we calculated the electronic structure including spin-orbit coupling, i.e., GGA $+U+$ SOC. We found a substantial orbital moment at the Ru site of around $0.033 \mu_{\mathrm{B}}$ at $U_{\text {eff }}=2 \mathrm{eV}$. We also calculated the magnetocrystalline anisotropy energy by aligning the spin quantization axis along [100], i.e., the chain direction and its perpendicular direction [001]. We found that this compound shows a strong easy axis [100] type of anisotropy ( $8.77 \mathrm{meV} /$ f.u.). Thus, the small value of the obtained Landé $g$ factor is due to the effect of a substantial orbital contribution toward the magnetic moment in addition to that of the spin part.

\section{DISCUSSION}

$\mathrm{Ag}_{2} \mathrm{RuO}_{4}$ and $\mathrm{Na}_{2} \mathrm{RuO}_{4}$ feature astoundingly similar crystal structures. The primary building blocks are virtually identical, even quantitatively with respect to bond lengths and angles. Moreover, the secondary building units are virtually the same: $\mathrm{RuO}_{3 / 1} \mathrm{O}_{2 / 2}$ chains that are similarly oriented, packed, and corrugated. Finally, the tertiary structures are alike, where sodium and silver form approximate hexagonal tubes arranged like honeycombs, centered by the Ru/O chains. Even the local connectivity modes of chains provided by $\mathrm{Ag}$ and $\mathrm{Na}$ are similar. Noteworthy, $\mathrm{Ag}$ is not in its preferred linear coordination; instead it displays, like $\mathrm{Na}$, a fivefold coordination.
The chains are arranged as pseudohexagonal rod packing in both structures, as shown in Figs. 4 and 5. Each chain, oriented in direction [100] for $\mathrm{Ag}_{2} \mathrm{RuO}_{4}$, respectively [010] in $\mathrm{Na}_{2} \mathrm{RuO}_{4}$ [45], is surrounded by six neighbor chains while two of those show the same orientation, in direction [001] for $\mathrm{Ag}_{2} \mathrm{RuO}_{4}$, respectively [101] for $\mathrm{Na}_{2} \mathrm{RuO}_{4}$. Yet, there is a topological difference: While the chains in $\mathrm{Ag}_{2} \mathrm{RuO}_{4}$ are undulated in the same direction [001], i.e., the chains $\left[\mathrm{Ru}-\mathrm{O}_{\text {axial }}\right]_{\infty}$ lay in the plane formed by the chain direction [100] and [001], i.e., (010) $)_{\text {ortho }}$, in $\mathrm{Na}_{2} \mathrm{RuO}_{4}$ the chains undulate in the plane $(100)_{\text {mono }}$, perpendicular rather than parallel to the direction in which chains look similar. Therefore, these "corrugation planes," unite close chains with two (though the two farthest) of their six neighbor chains in $\mathrm{Ag}_{2} \mathrm{RuO}_{4}$, whereas in $\mathrm{Na}_{2} \mathrm{RuO}_{4}$ they unite chains with two of the next-closest neighbors beyond the six closest ones (and, on top of that, with chains not containing the same symmetryequivalent $\mathrm{Ru}$ ions). In both cases, the equatorial $\mathrm{RuO}_{3}$ planes are perpendicular to that undulation (or corrugation) plane.

In terms of magnetic ordering, the two compounds differ even more significantly, see Fig. S6 and S9 [56]. In both cases the magnetic moment vectors lie in the equatorial $\mathrm{RuO}_{3}$ planes. In $\mathrm{Ag}_{2} \mathrm{RuO}_{4}$, in the corrugation plane (010) ortho $_{\text {, while }}$ in $\mathrm{Na}_{2} \mathrm{RuO}_{4}$ they are perpendicular to the corresponding plane, (100) mono. In $\mathrm{Ag}_{2} \mathrm{RuO}_{4}$ this corrugation plane is also the plane formed by equivalent next-neighbor chains. Chains inside these corrugation planes couple exclusively ferromagnetically.

Whereas in $\mathrm{Na}_{2} \mathrm{RuO}_{4}$ all magnetic moments are (nearly) parallel to each other, antiparallel between intrachain neighbors, and FM to next interchain neighbors, the magnetic moments in $\mathrm{Ag}_{2} \mathrm{RuO}_{4}$ are significantly inclined to each other, due to the topological difference of being parallel to the corrugation plane rather than perpendicular, while in both cases they are lying inside the equatorial $\mathrm{RuO}_{3}$ planes.

Obviously, the subtle topological differences in the interchain arrangement could hold for explaining the rather different magnetic ordering schemes. However, as we expect the interchain interaction to be small, we shall have a close look at intrachain bond lengths and angles. 
In $\mathrm{Na}_{2} \mathrm{RuO}_{4}$ we have $\mathrm{Ru}-\mathrm{Ru}$ intrachain distances of $3.506 \AA$; distances to next-nearest neighbors are 5.315, 5.339, 5.418, and 5.464 $\AA$ plus twice 5.418 $\mathrm{A}$ for $\operatorname{Ru}(1)$ and $5.506 \AA$ for $\mathrm{Ru}(2)$. Equatorial $\mathrm{Ru}-\mathrm{O}$ distances range from 1.753 to $1.782 \AA$; axial ones range from 1.974 to $2.000 \AA$. The O$\mathrm{Ru}-\mathrm{O}$ angle is $179.0^{\circ}$ or $178.3^{\circ}$. The angle of the axis and the plane is $86.4^{\circ}$ or $94.9^{\circ}$; the $\mathrm{O}-\mathrm{Ru}-\mathrm{O}$ angles in the plane range from $115.6^{\circ}$ to $123.2^{\circ}$. The intrachain $\mathrm{Ru}-\mathrm{O}-\mathrm{Ru}$ angles are $124.1^{\circ}$ or $122.7^{\circ}$. In $\mathrm{Ag}_{2} \mathrm{RuO}_{4}$ we find $\mathrm{Ru}-\mathrm{Ru}$ intrachain distances of 3.543(2) $\AA$; distances to next neighbors are twice 5.516(2) $\AA$ and four times 5.406(1) $\AA$. Equatorial Ru-O distances are 1.767(3) $\AA$ and twice 1.776(2) $\AA$; axial ones are 1.978(3) and 2.014(3) $\AA$. The O-Ru-O angle is $179.9(2)^{\circ}$. The angle of the axis and the plane $\mathrm{Ru}-\mathrm{O}$ bonds range from $85.8(2)^{\circ}$ to $94.1(2)^{\circ}$; the $\mathrm{O}-\mathrm{Ru}-\mathrm{O}$ angles in the plane are twice $117.9(2)^{\circ}$ to $124.2(2)^{\circ}$. The intrachain $\mathrm{Ru}-\mathrm{O}-\mathrm{Ru}$ angles are 125.1(1) .

The bipyramids are equally distorted, the interchain distances are similar, and the intrachain corrugation angle $\mathrm{Ru}-\mathrm{O}-$ $\mathrm{Ru}$ is larger by $0.8(8) \%$, respectively $2.0(8) \%$, but insignificantly. Only the $\mathrm{Ru}-\mathrm{Ru}$ intrachain distance is significantly longer by $1.05(5) \%$ in $\mathrm{Ag}_{2} \mathrm{RuO}_{4}$.

This longer intrachain $\mathrm{Ru}-\mathrm{Ru}$ distance despite similar bond distances and angles becomes obvious when regarding the connectivity between two coordination bipyramids in detail, as shown in Fig. S10 [56]. With the higher symmetry of $\mathrm{Ag}_{2} \mathrm{RuO}_{4}$, together with the $3 \%$ larger unit-cell volume, apart from the "corrugation" angle $\mathrm{Ru}-\mathrm{O}-\mathrm{Ru}$ of $125^{\circ}$, the coordination bipyramids are perfectly "staggered," i.e., an edge of one of the trigonal base planes (O3) is superposing a corner $(\mathrm{O} 2)$ of a neighboring one. As a consequence, the oxygen positions forming this corner $(\mathrm{O} 2)$ and this edge $(\mathrm{O} 3)$ come with the corrugation angle so close on one side that they form a tetrahedron together with the oxygen position (O1) which links the two bipyramids. Due to the corrugation and the repulsion of the oxygen atoms forming the empty tetrahedron, the trigonal base plane of the stretched bipyramid is-in opposite direction to the magnetic moment, pointing out of a plane perpendicular to the axis by $2.3^{\circ}$ —not exactly perpendicular to the bipyramid axis but inclined by $3.6^{\circ}$ to a plane perpendicular to the axis. In total, the moment is inclined by $5.9^{\circ}$ with respect to the plane spanned by $\mathrm{O} 2$ and $\mathrm{O} 3$. The $\mathrm{O}-\mathrm{O}$ distances in the trigonal plane are $2 \times$ 3.064(2) $\AA$ for O2-O3 and 3.135(2) $\AA$ for O3-O3, the latter constituting the common edge with the empty tetrahedron. The O-O distances from the trigonal plane to the apical O1 position are 2.573(2) $\AA$ for O1-O2 and $2 \times 2.634(2) \AA$ for O1-O3 not shared with the empty tetrahedron and 2.743(2) $\AA$ for $\mathrm{O} 1-\mathrm{O} 2$ and $2 \times 2.709$ (2) $\AA$ for $\mathrm{O} 1-\mathrm{O} 3$ shared with the tetrahedron, i.e., the longer bonds. The O-O distances in the empty tetrahedron are $2 \times 3.031$ (2) $\AA$ for O2-O3 (i.e., shorter than the $\mathrm{O} 2-\mathrm{O} 3$ distance of the bipyramid), in addition to the four distances evoked before, shared with the bipyramids. Shortest interchain O-O distances are 3.168(2) $\AA$ for O1-O2, $2 \times 3.125(1) \AA$ for O2-O3, and 3.218(2) $\AA$ for O3-O3.

In $\mathrm{Na}_{2} \mathrm{RuO}_{4}$ the bipyramids are not staggered that "precisely" but "squeezed" into a less symmetric nearly staggered conformation due to a lower unit-cell volume and incompressible $\mathrm{Ru}-\mathrm{O}$ bond lengths. One may speculate here, whether the nuclear structure of $\mathrm{Ag}_{2} \mathrm{RuO}_{4}$ would transform to a similar lower symmetry when high pressure is applied, and with it the magnetic ordering.

It appears that the magnetic moment has two possible orientations, both roughly in the trigonal base plane: either pointing into the direction of the oxygen position forming the plane which is not part of the shared empty tetrahedron between bipyramids, as in the case of $\mathrm{Ag}_{2} \mathrm{RuO}_{4}$, or roughly perpendicular to this, parallel to the edge of the trigonal plane shared with the empty tetrahedron, as in the case of $\mathrm{Na}_{2} \mathrm{RuO}_{4}$. The latter results in a perfect AFM ordering scheme inside each chain, whereas the former leaves each chain with a consequence of the resulting magnetic moment to be compensated only by the perfect AFM arrangement of layers of FM ordered chains (Fig. S9 [56]).

\section{CONCLUSIONS}

$\mathrm{Ag}_{2} \mathrm{RuO}_{4}$ displays a trigonal bipyramidal coordination by oxygen for $\mathrm{Ru}^{6+}$, which is rare in transition-metal oxide chemistry. These local building units are linked to form an effective infinite 1D quantum spin-chain system with $S=$ 1. The oxoruthenate chains are arranged as an approximate hexagonal rod packing, significantly deviating from ideal trigonal symmetry, thus damping frustration effects commonly encountered with magnetic exchange on trigonal real space structures. As a consequence, this structural symmetry breaking results in a complex variety of electronic and magnetic interaction paths. While the intrachain coupling is clearly FM, the interchain couplings are either AFM or FM, sensitively depending on slight structural differences in mutual arrangements of and separations between the chains. However, the overall magnetic ordering is predominantly of AFM type. Although the title compound and sodium analog, $\mathrm{Na}_{2} \mathrm{RuO}_{4}$, are very similar with respect to their crystal structure and valence electronic configuration, it comes as a surprise that the magnetic ordering schemes developed are substantially different, starting with the strongest intrachain coupling, which is AFM in $\mathrm{Na}_{2} \mathrm{RuO}_{4}$, while all interchain exchange paths are FM in nature. Thus, the results obtained on the title compound, and direct comparison with very similar $\mathrm{Na}_{2} \mathrm{RuO}_{4}$, are suited to demonstrate the effect on magnetic exchange couplings of only very gentle variations in the geometric detailing of the structures hosting interacting magnetic species.

\section{ACKNOWLEDGMENTS}

The authors acknowledge Dr. Walter Schnelle and Mr. Ralf Koban for magnetic, heat capacity, and resistivity measurements. S.K. thanks Department of Science \& Technology, Government of India, for providing an INSPIRE faculty research grant (Grant No. DST/INSPIRE/04/2016/000431) and IUAC-New Delhi, India for providing computational facilities. S.S. thanks IIT Goa, Ministry of Human Resource Development, Government of India for providing a research fellowship. 
[1] A. Vasiliev, O. Volkova, E. Zvereva, and M. Markina, npj Quantum Mater. 3, 18 (2018).

[2] F. D. M. Haldane, Phys. Rev. Lett. 50, 1153 (1983).

[3] Z. Honda, H. Asakawa, and K. Katsumata, Phys. Rev. Lett. 81, 2566 (1998).

[4] D. C. Johnston, R. K. Kremer, M. Troyer, X. Wang, A. Klümper, S. L. Bud'ko, A. F. Panchula, and P. C. Canfield, Phys. Rev. B 61, 9558 (2000).

[5] S. Sachdev, Quantum Phase Transitions (Cambridge University, New York, 1999).

[6] P. Lemmens, G. Güntherodt, and C. Gros, Phys. Rep. 375, 1 (2003).

[7] M. Hase, I. Terasaki, and K. Uchinokura, Phys. Rev. Lett. 70, 3651 (1993).

[8] S. Yamashita, Y. Nakazawa, M. Oguni, Y. Oshima, H. Nojiri, Y. Shimizu, K. Miyagawa, and K. Kanoda, Nat. Phys. 4, 459 (2008).

[9] M. Yamashita, N. Nakata, Y. Kasahara, T. Sasaki, N. Yoneyama, N. Kobayashi, S. Fujimoto, T. Shibauchi, and Y. Matsuda, Nat. Phys. 5, 44 (2009).

[10] C. Balz, B. Lake, J. Reuther, H. Luetkens, R. Schönemann, T. Herrmannsdörfer, Y. Singh, A. T. M. N Islam, E. M. Wheeler, A. Rodriguez-Rivera Jose, T. Guidi, G. Simeoni Giovanna, C. Baines, and H. Ryll, Nat. Phys. 12, 942 (2016).

[11] G. Jackeli and G. Khaliullin, Phys. Rev. Lett. 102, 017205 (2009).

[12] J. Chaloupka, G. Jackeli, and G. Khaliullin, Phys. Rev. Lett. 105, 027204 (2010).

[13] B. J. Kim, H. Ohsumi, T. Komesu, S. Sakai, T. Morita, H. Takagi, and T. Arima, Science 323, 1329 (2009).

[14] B. J. Kim, H. Jin, S. J. Moon, J. Y. Kim, B. G. Park, C. S. Leem, J. Yu, T. W. Noh, C. Kim, S. J. Oh, J. H. Park, V. Durairaj, G. Cao, and E. Rotenberg, Phys. Rev. Lett. 101, 076402 (2008).

[15] L. F. Mattheiss, Phys. Rev. B 13, 2433 (1976).

[16] S. Kanungo, R. Datta, and S. K. Panda, and T. Saha-Dasgupta, J. Phys.: Condens. Matter 25, 505503 (2013).

[17] E. Jakobi, S. Kanungo, S. Sarkar, S. Schmitt, and T. SahaDasgupta, Phys. Rev. B 83, 041103(R) (2011).

[18] G. Cao, S. McCall, M. Shepard, J. E. Crow, and R. P. Guertin, Phys. Rev. B 56, 321 (1997).

[19] J. J. Randall and R. Ward, J. Am. Chem. Soc. 81, 2629 (1959).

[20] L. Klein, J. S. Dodge, C. H. Ahn, J. W. Reiner, L. Mieville, T. H. Geballe, M. R. Beasley, and A. Kapitulnik, J. Phys.: Condens. Matter 8, 10111 (1996)

[21] J. M. Longo and P. M. Raccah, and J. B. Goodenough, J. Appl. Phys. 39, 1327 (1968).

[22] A. P. Mackenzie and Y. Maeno, Rev. Mod. Phys. 75, 657 (2003).

[23] K. W. Plumb, J. P. Clancy, L. J. Sandilands, V. V. Shankar, Y. F. Hu, K. S. Burch, H.-Y. Kee, and Y.-J. Kim, Phys. Rev. B 90, 041112(R) (2014).

[24] A. Banerjee, C. A. Bridges, J. Q. Yan, A. A. Aczel, L. Li, M. B. Stone, G. E. Granroth, M. D. Lumsden, Y. Yiu, J. Knolle, S. Bhattacharjee, D. L. Kovrizhin, R. Moessner, D. A. Tennant, D. G. Mandrus, and S. E. Nagler, Nat. Mater. 15, 733 (2016).

[25] A. Alexander, P. D. Battle, J. C. Burley, D. J. Gallon, C. P. Grey, and S. H. Kim, J. Mater. Chem. 13, 2612 (2003).

[26] W-J Son, P. Manuel, D. Adroja, and M-H. Whangbo, Inorg. Chem. 50, 9400 (2011).

[27] M. D. Johannes, A. M. Stux, and K. E. Swider-Lyons, Phys. Rev. B 77, 075124 (2008).
[28] Y. Miura, Y. Yasui, M. Sato, N. Igawa, and K. Kakurai, J. Phys. Soc. Jpn. 76, 033705 (2007).

[29] K. Hoang, M. Oh, and Y. Choi, ACS Appl. Electron. Mater. 1, 75 (2019).

[30] H. Lux, Z. Electrochem. 45, 303 (1939).

[31] H. Flood and T. Forland, Acta Chem. Scand. 1, 592 (1947).

[32] C. Linke and M. Jansen, Z. Anorg. Allg. Chem. 623, 1441 (1997).

[33] M. Jansen, J. Less Common Met. 76, 285 (1980).

[34] M. Jansen, Angew. Chem. Int. Ed. 26, 1098 (1987).

[35] H. Schmidbaur and A. Schier, Angew. Chem. Int. Ed. 54, 746 (2015).

[36] B. U. Köhler, M. Jansen, and W. Weppner, J. Solid State Chem. 57, 227 (1985).

[37] C. Friebel and M. Jansen, Z. Naturforsch. B 39, 739 (1984).

[38] B. E. Prasad, S. Kanungo, M. Jansen, A. C. Komarek, B. Yan, P. Manuel, and C. Felser, Chemistry-A European Journal 23, 4680 (2017).

[39] W. Klein and M. Jansen, Acta Crystallogr. C 61, i1 (2005).

[40] B. E. Prasad, P. Kazin, A. C. Komarek, C. Felser, and M. Jansen, Angew. Chem. Int. Ed. 55, 4467 (2016).

[41] J. Darriet and J Galy, Bull. Soc. Fr. Mineral. Cristallogr. 97, 3 (1974).

[42] M. Drillon, J. Darriet, and R. Georges, J. Phys. Chem. Solids 38, 411 (1977).

[43] J. T. Haraldsen, M. B. Stone, M. D. Lumsden, T. Barnes, R. Jin, J. W. Taylor, and F. Fernandez-Alonso, J. Phys.: Condens. Matter 21, 506003 (2009).

[44] K. M. Mogare, K. Friese, W. Klein, and M. Jansen, Z. Anorg. Allg. Chem. 630, 547 (2004).

[45] K. M. Mogare, D. V. Sheptyakov, R. Bircher, H-U. Güdel, and M. Jansen, Eur. Phys. J. B 52, 371 (2006).

[46] M. Shikano, R. K. Kremer, M. Ahrens, H. J. Koo, M. H. Whangbo, and J. Darriet, Inorg. Chem. 43, 5 (2004).

[47] A. Balodhi and Y. Singh, Phys. Rev. Mater. 2, 024403 (2018).

[48] G. Nowogrocki, Sur le comportement en solution aqueuse des valences supérieures du ruthénium, Thèse Docteur ès Sciences, Université Lille1 (1967).

[49] Bruker AXS, TOPAS, Version 4.2 (Bruker AXS Inc., Madison, WI, 2009).

[50] T. C. Hansen, P. F. Henry, H. E. Fischer, J. Torregrossa, and P. Convert, Meas. Sci. Technol. 19, 034001 (2008).

[51] G. Kresse and J. Hafner, Phys. Rev. B 47, 558 (1993).

[52] G. Kresse and J. Furthmüller, Phys. Rev. B 54, 11169 (1996).

[53] J. P. Perdew, K. Burke, and M. Ernzerhof, Phys. Rev. Lett. 77, 3865 (1996).

[54] V. I. Anisimov, I. V. Solovyev, M. A. Korotin, M. T. Czyżyk, and G. A. Sawatzky, Phys. Rev. B 48, 16929 (1993).

[55] S. L. Dudarev, G. A. Botton, S. Y. Savrasov, C. J. Humphreys, and A. P. Sutton, Phys. Rev. B 57, 1505 (1998).

[56] See Supplemental Material at http://link.aps.org/supplemental/ 10.1103/PhysRevMaterials.4.024418 for TGA/DTA data, susceptibility data, electrical resistivity data, structure plots, neutron-diffraction patterns taken at a wavelength of $2.41 \AA$ at 1.8 and $76 \mathrm{~K}$, projection of the magnetic structure of $\mathrm{Na}_{2} \mathrm{RuO}_{4}$, lattice constants and unit-cell volume as a function of temperature with Einstein model fit above $T_{\mathrm{N}}$, plot of the volume strain as a function of the squared magnetic moment, and table of atomic parameters.

[57] M. Hillert and M. Jarl, Calphad 2, 227 (1978). 
[58] T. Roisnel and J. Rodríquez-Carvajal, Mater. Sci. Forum 378381, 118 (2001).

[59] J. Rodríguez-Carvajal, IUCR-CPD Newsl. 26, 12 (2001).

[60] I. S. Anderson, P. J. Brown, J. M. Carpenter, G. Lander, R. Pynn, J. M. Rowe, O. Schärpf, V. F. Sears, and B. T. M. Willis, Neutron Techniques International Tables for Crystallography Mathematical, Physical and Chemical Tables Vol. C (International Union of Crystallography, Chester, England, 2006), pp. 430-87.

[61] T. Chatterji and T. C. Hansen, J. Phys.: Condens. Matter 23, 276007 (2011).

[62] R. L. Carlin, Magnetochemistry (Springer-Verlag, Berlin, 1986).

[63] M. E. Fisher, Am. J. Phys. 32, 343 (1964).

[64] O. Kahn, Molecular Magnetism (VCH, New York, 1993).

[65] A. N. Vasiliev, O. L. Ignatchik, M. Isobe, and Y. Ueda, Phys. Rev. B 70, 132415 (2004).

[66] H. T. Dang, J. Mravlje, A. Georges, and A. J. Millis, Phys. Rev. B 91, 195149 (2015).

[67] J. Mravlje, M. Aichhorn, T. Miyake, K. Haule, G. Kotliar, and A. Georges, Phys. Rev. Lett. 106, 096401 (2011).

[68] Z. V. Pchelkina, I. A. Nekrasov, Th. Pruschke, A. Sekiyama, S. Suga, V. I. Anisimov, and D. Vollhardt, Phys. Rev. B 75, 035122 (2007).
[69] S. Ryee and M. J. Han, Sci. Rep. 8, 9559 (2018).

[70] M. H.-E. Musa Saad, Mater. Chem. Phys. 204, 350 (2018).

[71] R. Bentata, S. Bentata, B. Bouadjemi, T. Lantri, and D. Chenine, Chin. J. Phys. 59, 28 (2019).

[72] C. Gauvin-Ndiaye, A.-M. S. Tremblay, and R. Nourafkan, Phys. Rev. B 99, 125110 (2019).

[73] K. I. Kugel and D. I. Khomskii, Zh. Eksp. Teor. Fiz. 64, 1429 (1973) [Sov. Phys. JETP 37, 725 (1973)].

[74] K. I. Kugel and D. I. Khomskii, Sov. Phys. Usp. 25, 231 (1982).

[75] V. V. Mazurenko, F. Mila, and V. I. Anisimov, Phys. Rev. B 73, 014418 (2006).

[76] C. S. Hellberg, W. E. Pickett, L. L. Boyer, H. T. Stokes, and M. J. Mehl, J. Phys. Soc. Jpn. 68, 3489 (1999).

[77] S. Kanungo, B. Yan, M. Jansen, and C. Felser, Phys. Rev. B 89, 214414 (2014).

[78] S. Kanungo, B. Yan, C. Felser, and M. Jansen, Phys. Rev. B 93, 161116(R) (2016).

[79] J. Sannigrahi, J. Sichelschmidt, B. Koo, A. Banerjee, S. Majumdar, and S. Kanungo, J. Phys.: Condens. Matter 31, 245802 (2019).

[80] J. B. Goodenough, Phys. Rev. 100, 564 (1955).

[81] J. B. Goodenough, Magnetism and the Chemical Bond (Interscience, New York, 1963). 